\title{
Hopf bifurcation with cubic symmetry and instability of ABC
}

flow.

\author{
Peter Ashwin ${ }^{a}$ and Olga Podvigina ${ }^{a-d}$ \\ ${ }^{a}$ School of Mathematical Sciences \\ University of Exeter \\ Exeter EX4 4QE, UK \\ ${ }^{b}$ Observatoire de la Côte d'Azur, CNRS UMR 6529, \\ BP 4229, 06304 Nice Cedex 4, France \\ ${ }^{c}$ International Institute of Earthquake Prediction Theory \\ and Mathematical Geophysics,
}

79 bldg. 2, Warshavskoe ave., 113556 Moscow, Russian Federation

${ }^{d}$ Laboratory of General Aerodynamics, Institute of Mechanics, Lomonosov Moscow State University, 1, Michurinsky ave., 119899 Moscow, Russian Federation

October 22, 2002

\begin{abstract}
We examine the dynamics of generic Hopf bifurcation in a system that is symmetric under the action of the rotational symmetries of the cube. We classify the generic branches of periodic solutions at bifurcation; there are generically 27 branches corresponding to maximal symmetries, organized into five symmetry types. There are also up to 22 periodic solution branches of two other symmetry types. These results are found by examination of the normal form (with $\mathbf{S}^{1}$ normal form symmetry) for the bifurcation truncated at the third order.

In addition to the periodic branches whose branching and stability we find, there are several branches of tori, homoclinic bifurcations and chaotic attractors in the dynamics of the third order normal form. Since many of these features are not amenable to analysis we give some numerical examples. On breaking the normal form symme-
\end{abstract}


try, there may be breakup of the branches of tori, but the predictions for the periodic solutions will be reliable.

For the Navier-Stokes equations with a particular forcing, an ABC flow is a dynamically stable solution for small Reynolds numbers $R$. For the most symmetric case, $A=B=C=1$, the first instability of this system is a Hopf bifurcation at $R=13.04$ with rotational symmetry of the cube. We use our normal form analysis to explain the observed behaviour of solutions at this primary instability. Numerical simulations show that there is supercritical branching to rotating waves that alternate between the three axes, which undergo secondary Hopf bifurcation to a 2-torus at approximately $R=13.09$. The eight symmetrically related tori break up and then merge to form a chaotic attractor with full symmetry. We can explain all these features by use of the generic third order normal form and $\mathbf{S}^{1}$ normal form symmetry breaking terms.

\section{Introduction}

The so-called ABC flow is a fully three-dimensional steady flow of incompressible fluid with velocity field

$$
\mathbf{u}_{A B C}=\left(A \sin x_{3}+C \cos x_{2}, B \sin x_{1}+A \cos x_{3}, C \sin x_{2}+B \cos x_{1}\right),
$$

where $A, B$ and $C$ are constants. This flow satisfies the Beltrami condition $\nabla \times \mathbf{u}_{A B C}=$ $\alpha \mathbf{u}_{A B C}$ with $\alpha=1$ and hence it is a solution to the Euler equations with the vanishing force. Arnold [2] has proved that a steady solution to a force-free Euler equation can have chaotic streamlines only if the solution possesses the Beltrami property; he introduced $\mathrm{ABC}$ flows as the simplest analytical example of vector fields satisfying this condition. As such, ABC flows have enjoyed a close attention of a large number of mathematicians and physisists. Hénon [29] showed numerically that for $A=\sqrt{3}, B=\sqrt{2}, C=1$ the $\mathrm{ABC}$ flow is indeed chaotic. The particle paths of a variety of ABC flows have been studied in [16]. If at least one coefficient in (1) vanishes, the flow is integrable. Using the Painlevé test, Dombre et al. [16] argued that this condition for integrability of an ABC flow is also necessary. The Poincaré sections computed for certain flows of that type revealed that the flows do have chaotic streamlines; indeed the largest Lyapunov exponents for streamlines of some ABC flows were computed in [18] and were found to be positive.

If a triangle can be constructed with sides equal to $A^{2}, B^{2}$ and $C^{2}$, the flow has 8 stagnation points in a periodicity cube; there are no stagnation points otherwise. In the 
case $A=B=C=1$ stagnation points are connected by heteroclinic trajectories, which are straight lines [12].

Non-integrability of streamlines, giving rise to chaotic mixing of a passive scalar within the flow, is a necessary condition for a flow to act as a fast magnetic field generator $[45,46,31]$. For this reason, and because heteroclinic structure of trajectories of ABC flows is rather well understood, they are widely employed in the study of magnetic field generation. In the context of the kinematic dynamo, $\mathrm{ABC}$ flows were first examined in [10]. It was shown that they can act as dynamos, e.g. see $[4,20,21,23,24,18,19,11]$. These computations suggest that for certain sets of coefficients the dynamos are fast. ABC flows were also proposed as prototypes for the study of the development of turbulence (see $[37,38,39])$. Magnetic field reversals were observed in an ABC-force driven fully non-linear MHD system [40].

Generically, (1) has the symmetry group isomorphic to $\mathbf{D}_{2}$ if we exclude time reversal symmetries (these are symmetries of the flow but not of the Navier-Stokes equations). If two coefficients are equal, it is isomorphic to $\mathbf{D}_{4}$. For $A=B=C$ the group, denoted by $\mathcal{H}$, has 24 elements and it is isomorphic to the rotation group of a cube [3],[16]. We consider the particular case:

$$
A=B=C=1 \text {. }
$$

For any Reynolds number $R, \mathbf{u}_{A B C}$ is a steady solution of the Navier-Stokes equation

$$
\frac{\partial \mathbf{v}}{\partial t}=\mathbf{v} \times(\nabla \times \mathbf{v})-\nabla p+\frac{1}{R} \Delta \mathbf{v}+\mathbf{f},
$$

subject to the incompressibility condition

$$
\nabla \cdot \mathbf{v}=0
$$

and the force

$$
\mathbf{f}=\frac{1}{R} \mathbf{u}_{A B C}
$$

( $p$ is the pressure). Space-periodic boundary conditions are assumed in $x_{1}, x_{2}, x_{3}$, namely

$$
\begin{aligned}
& \mathbf{v}\left(x_{1}, x_{2}, x_{3}\right)=\mathbf{v}\left(x_{1}+2 k \pi, x_{2}+2 l \pi, x_{3}+2 m \pi\right) \\
& p\left(x_{1}, x_{2}, x_{3},\right)=p\left(x_{1}+2 k \pi, x_{2}+2 l \pi, x_{3}+2 m \pi\right)
\end{aligned}
$$

for any $(k, l, m) \in \mathbf{Z}^{3}$. In this setup, any symmetry of the ABC flow is also a symmetry of the equation, but solutions that branch from the $\mathrm{ABC}$ flow solution may break some or all of these symmetries. 
One of the aims of this paper is to examine the primary dynamical instability of this flow on increasing Reynolds number by showing that its stable dynamics near this instability are governed by a generic Hopf bifurcation with the appropriate symmetry. Using this we predict and find the existence of many different branches of periodic solutions as well as more exotic dynamics.

Numerical results on bifurcations of time-dependent solutions of the Navier-Stokes equation (1-5) for $R \leq 50$ were presented in [37]-[39]. It can be proven that for $R<0.5$ the flow (1-5) is a unique steady state of $(3,4)$, and it is stable. Computations show that the flow is stable for $R \leq 13.04$ [22],[37] and that it is a unique attractor of this hydrodynamical system for $R \leq 7.8$ [37]. The trivial steady state - the 1:1:1 ABC flow $(1,2)$ - becomes unstable in a Hopf bifurcation at some $R=R_{0}$, where $R_{0}$ can be numerically located at approximately 13.044 [39]. The action of the group $\mathcal{H}$ on the center eigenspace at this bifurcation is isomorphic to the standard representation of the group of rotational symmetries of the cube, $\mathbf{O}$ (see [38]).

In this paper we firstly characterize generic Hopf bifurcation with the symmetry $\mathbf{O}$. Secondly, we analyze this instability and secondary bifurcations in the particular case of instability of $\mathbf{u}_{A B C}$ in the above problem at $R=R_{0}$ to perturbations in the Eulerian velocity field.

In order to do this we apply methods and results from generic equivariant bifurcation theory to this problem; see $[25,26]$ for discussion and development of methods, details of the theory and many examples. An important feature of this theory is that a generic Hopf bifurcation of a symmetric equilibrium will (in the absence of further degeneracies) occur within a linear subspace that is one of the $\mathbf{C}$-irreducible representations (henceforth called irreps) of the symmetry group. The group $\mathbf{O}=S_{4}$ of permutations of 4 elements has five C-irreps $W_{0}, \cdots W_{4}$; two of these are on $\mathbf{C}^{3}$, one on $\mathbf{C}^{2}$ and two on $\mathbf{C}$. We consider only bifurcations for representation $W_{0}$ on $\mathbf{C}^{3}$ where $\mathbf{O}$ acts purely by rotations. This irrep is two copies of the $\mathbf{R}$-irrep on $\mathbf{R}^{3}$ given by rotational symmetries of a cube. The other $\mathbf{R}$-irrep on $\mathbf{R}^{3}$ is the set of symmetries of a regular tetrahedron acting by permuting its vertices; note that this includes reflections and has no rotations of order four (but it does have a rotation-reflection of order four).

There exists a nontrivial isomorphism of the group $\mathbf{O} \times \mathbf{S}^{1}$ into itself. We will give an explicit form for it in Section 2. The action of $\mathbf{O} \times \mathbf{S}^{1}$ on $W_{1}$ (the $\mathbf{C}^{3}$ representation of 
$\mathbf{O} \times \mathbf{S}^{1}$ distinct from $W_{0}$ ) can be represented as a composition of the isomorphism and the action of the group on $W_{0}$. Thus all our results concerning bifurcations with symmetry group $\mathbf{O} \times \mathbf{S}^{1}$ on $W_{0}$ are also applicable to the representation $W_{1}$, the isomorphism being taken into account. (However, representations of the group $\mathbf{O}$ on $W_{0}$ and $W_{1}$ are not related by an automorphism of the group, and so the bifurcations on $W_{0}$ and $W_{1}$ are different in this sense.)

Generic steady state bifurcations with several types of cubic symmetry have been investigated by many authors. In particular, bifurcations on cubic lattices are studied in [8]. An analysis of steady state bifurcation with the 48 element group Õ of rotations and reflections of the cube is considered in [35]. Some work has been done on steady bifurcation with another element subgroup of $\tilde{\mathbf{O}}$ of elements that preserves the ordering of the axes; in particular [27] uses the third order normal form to show that branches of robust attracting heteroclinic cycles can arise as a generic possibility. However, this group is not isomorphic to $\mathbf{O}$; it has no elements of order four. As to Hopf bifurcations, an analysis of generic Hopf bifurcation on square (resp. cubic) lattices was carried out in [42] (resp. [14]). Barany and Swift [44] examine generic Hopf bifurcation with the symmetry of an index 2 subgroup of $\mathbf{O}$; they find branches of robust homoclinic orbits branching from the trivial solution for an open and dense set of normal form coefficients (these homoclinic orbits were further investigated in [6]).

For the action of $\mathbf{O} \times \mathbf{S}^{1}$ on $\mathbf{C}^{3}$ that we consider here, there are two families of fixed point subspaces that are isomorphic to $\mathbf{C}^{2}$. In one of these we can apply the analysis of the Hopf bifurcation with dihedral $\mathbf{D}_{4}$ symmetry of Swift [43] to find branches of periodic solutions with submaximal symmetries. The other subspace also supports submaximal symmetry periodic solutions.

The structure of the paper is as follows: In Section 2 we characterize the group $\mathbf{O}$, discuss its representation on $\mathbf{R}^{3}$ by rotations and the representation it induces on $\mathbf{C}^{3}$ at a generic Hopf bifurcation. For this representation we discuss the isotropy subgroups and invariant subspaces and derive the general normal form at bifurcation. Much of the dynamics we investigate here is determined by the normal form truncated at third order. We classify the generic branching behaviour and stabilities of these periodic orbits at bifurcation in terms of the coefficients of this normal form. There are five families of periodic solutions with maximal symmetries (all maximal symmetries are $\mathbf{C}$-axial in 
the terms of [15]). These solutions are always present; two additional families (with submaximal symmetries) are present for an open (but not dense) set of normal form coefficients. All these periodic solutions have frequencies close to the Hopf frequency. Since all branching periodic solutions are determined at third order for an open set of normal form parameters, Theorem 11.2 in [26] implies that the branching of periodic solutions of the full equations are generically determined by the third order normal form.

In addition to periodic branching behaviour, other branches can occur in the normal form to chaotic attractors and quasiperiodic branches (for an open set of normal form coefficients) and homoclinic/heteroclinic cycles (for a codimension one set of normal form coefficients). In Section 3 some indicative examples of this are investigated numerically by path-following, as a complete analytical classification of all possible attractors is not presently possible. We also discuss the effect of breaking the normal form symmetry introduced at Hopf bifurcation by virtue of the fact that all terms that do not commute with the $\mathbf{S}^{1}$ symmetry of phase shifts given by solution of the linear equations can be transformed away to arbitrarily high order. These 'flat terms' can however cause dynamically important effects such as torus breakup; we give some examples.

In Section 4 we return to the particular case of instability of the ABC flow, describing and discussing attractors observed numerically at the bifurcation. We discuss how the observed transition to a chaotic attractor with full symmetry on increasing $R$ can be explained by the normal form model analysed in Sections 2 and 3 . The branches of periodic solutions correspond to time-periodic Eulerian solutions close to the original ABC flow; moreover, the quasiperiodic and chaotic solutions are also close to the original $\mathrm{ABC}$ flow and can be thought of as its time-dependent perturbations. Finally, some limitations, the effects of symmetry breaking, and possible extensions of this present work are briefly discussed in Section 5.

\section{Hopf bifurcation with rotational symmetry of the cube.}

The group We consider Hopf bifurcation with cubic symmetry for the complex irreducible action on $\mathbf{C}^{3}$ of the group $\mathbf{O} \times \mathbf{S}^{1}$ generated by the three-fold rotation

$$
\rho_{111}:\left(z_{1}, z_{2}, z_{3}\right) \mapsto\left(z_{2}, z_{3}, z_{1}\right)
$$


the four-fold rotation

$$
\rho_{001}:\left(z_{1}, z_{2}, z_{3}\right) \mapsto\left(z_{2},-z_{1}, z_{3}\right)
$$

and the normal form $\mathbf{S}^{1}$ phase shift symmetry

$$
\gamma_{\theta}:\left(z_{1}, z_{2}, z_{3}\right) \mapsto e^{i \theta}\left(z_{1}, z_{2}, z_{3}\right)
$$

We refer to the group acting in this way as $\mathbf{O} \times \mathbf{S}^{1}$ (the representation on $W_{0}$ of the introduction). We denote

$$
\kappa_{110}^{+}:\left(z_{1}, z_{2}, z_{3}\right) \mapsto\left(z_{2}, z_{1},-z_{3}\right), \quad \kappa_{110}^{-}:\left(z_{1}, z_{2}, z_{3}\right) \mapsto\left(-z_{2},-z_{1},-z_{3}\right), \text { etc. }
$$

Note that $\kappa_{110}^{+}=\rho_{111}^{2} \rho_{001} \rho_{111}^{2}$ and $\kappa_{110}^{-}=\rho_{001}^{2} \kappa_{110}^{+}$. The symmetry $\rho_{111}$ has order three and corresponds to a rotation around a vertex of the cube, $\rho_{001}$ has order four and corresponds to a rotation about the centre of a cube face by one quarter of a turn, $\kappa_{110}^{+}$has order two and corresponds to a rotation about a line through midpoints of opposite edges of the cube by a half of a turn. We refer to the (conjugacy class of the) group generated by $\kappa_{011}^{-} \gamma_{\pi}$ as $\mathbf{Z}_{2}(e)$ and to the one generated by $\rho_{001}^{2} \gamma_{\pi}$ as $\mathbf{Z}_{2}(f)$. The $\gamma$ act as temporal phase-shift symmetries that are present in the normal form but in fact these symmetries are broken in generic problems by high order terms. The isomorphism of the group $\mathbf{O} \times \mathbf{S}^{1}$ to itself that relates the $\mathbf{C}^{3}$ representations $W_{0}$ and $W_{1}$ is given by:

$$
\rho_{111} \mapsto \rho_{111}, \quad \rho_{001} \mapsto \gamma_{\pi} \rho_{001}, \quad \gamma_{\theta} \mapsto \gamma_{\theta}
$$

Invariant subspaces For an action of a group $G$, the isotropy subgroup of a point $x$ is the largest subgroup of $G$ that fixes that point. Given any subgroup $H$ the fixed point space $\operatorname{Fix}(H)$ is the set of points fixed by all group elements in $H$ (see e.g. [26]).

The isotropy subgroups and invariant subspaces for the irreducible action of $\mathbf{O} \times \mathbf{S}^{1}$ on $\mathbf{C}^{3}$ are listed in Table 1 with a typical point, dimension, generators, number of conjugates and normalizers tabulated. Note that all nontrivial isotropy subgroups contain mixed spatio-temporal symmetries; often these are differentiated from purely spatial symmetries by addition of a tilde, but in this case it is unnecessary. This is similar to the case for $\mathbf{D}_{4}$ Hopf bifurcation where the action of $\mathbf{D}_{4} \times \mathbf{S}^{1}$ on $\mathbf{C}^{2}$ has non-trivial kernel. Recall that if $H$ is an isotropy subgroup then the normalizer $\operatorname{Norm}(H)=\left\{g \in \mathbf{O} \times \mathbf{S}^{1}: g H=H g\right\}$ is the largest subgroup that maps $\operatorname{Fix}(H)$ to itself. The isotropy subgroups can be partially ordered into the lattice shown in Figure 1 by considering containment (up to conjugacy). 


\begin{tabular}{c|crccc} 
Name & Typical point & $\operatorname{dim}_{\mathbf{C}}$ & Generators & Conj. & Norm. \\
\hline $\mathbf{O} \times \mathbf{S}^{1}$ & $(0,0,0)$ & 0 & $\left\{\rho_{111}, \rho_{100}, \gamma_{\theta}\right\}$ & 1 & $\mathbf{O} \times \mathbf{S}^{1}$ \\
$\mathbf{D}_{3}$ & $(z, z, z)$ & 1 & $\left\{\rho_{111}, \kappa_{110}^{-} \gamma_{\pi}\right\}$ & 4 & $\mathbf{D}_{3} \times \mathbf{S}^{1}$ \\
$\mathbf{D}_{2}$ & $(z, z, 0)$ & 1 & $\left\{\kappa_{110}^{+}, \kappa_{110}^{-} \gamma_{\pi}\right\}$ & 6 & $\mathbf{D}_{2} \times \mathbf{S}^{1}$ \\
$\mathbf{D}_{4}$ & $(z, 0,0)$ & 1 & $\left\{\rho_{100}, \rho_{001}^{2} \gamma_{\pi}\right\}$ & 3 & $\mathbf{D}_{4} \times \mathbf{S}^{1}$ \\
$\mathbf{Z}_{4}$ & $(z, i z, 0)$ & 1 & $\left\{\rho_{001} \gamma_{\pi / 2}\right\}$ & 6 & $\mathbf{Z}_{4} \times \mathbf{S}^{1}$ \\
$\mathbf{Z}_{3}$ & $\left(z, z e^{2 \pi i / 3}, z e^{4 \pi i / 3}\right)$ & 1 & $\left\{\rho_{111} \gamma_{2 \pi / 3}\right\}$ & 8 & $\mathbf{Z}_{3} \times \mathbf{S}^{1}$ \\
$\mathbf{Z}_{2}(f)$ & $\left(z_{1}, z_{2}, 0\right)$ & 2 & $\left\{\rho_{001}^{2} \gamma_{\pi}\right\}$ & 3 & $\mathbf{D}_{4} \times \mathbf{S}^{1}$ \\
$\mathbf{Z}_{2}(e)$ & $\left(z_{1}, z_{2}, z_{2}\right)$ & 2 & $\left\{\kappa_{011}^{-} \gamma_{\pi}\right\}$ & 6 & $\mathbf{D}_{2} \times \mathbf{S}^{1}$ \\
$\mathbf{1}$ & $\left(z_{1}, z_{2}, z_{3}\right)$ & 3 & $\{e\}$ & 1 & $\mathbf{O} \times \mathbf{S}^{1}$
\end{tabular}

Table 1: Table of the isotropy subgroups for the action of $\mathbf{O} \times \mathbf{S}^{1}$ on $\mathbf{C}^{3}$ considered here. There are nine possible symmetry types; the number of conjugate subgroups and the normalizer are listed as well as the dimension of the fixed point subspace and a list of generators. The suffices (e) and (f) refer to edge and face symmetries respectively.

Note that there is a candidate for a robust (relative) heteroclinic cycle that connects equilibria in the $\mathbf{D}_{2}$ and $\mathbf{D}_{4}$ subspaces and passes through $\mathbf{Z}_{2}(e)$ and $\mathbf{Z}_{2}(f)$. This setup is not present in the $\mathbf{D}_{4}$ Hopf bifurcation and is a truly $3 \mathrm{D}$ phenomenon. We have not managed to rule out such robust heteroclinic cycles but neither have we observed them in the normal form dynamics.

One can demonstrate that there are no robust homoclinic cycles for this group action, by noting that the only chains of properly contained subgroups $1<K<H<\mathbf{O} \times \mathbf{S}^{1}$ have $K=\mathbf{Z}_{2}(e)$ or $\mathbf{Z}_{2}(f)$, and in both cases there is no $g$ and $H$ such that $\operatorname{Norm}(K) \cap g H=\emptyset$ and $K<H \cap H^{g}$. Hence by Proposition 2.3 in [7] there can be no such robust homoclinic cycles (although there may still be robust heteroclinic cycles).

Normal form The general formal normal form for a vector field with the given action $\mathbf{O} \times \mathbf{S}^{1}$ symmetry can be written as follows:

$$
\dot{z_{1}}=\sum_{\left(l_{1}, l_{2}, q_{1}, q_{2}, q_{3}\right) \in I^{\prime}} C_{l_{1}, l_{2}, q_{1}, q_{2}, q_{3}}^{1} z_{1}^{l_{1}}\left|z_{1}\right|^{2 q_{1}}\left(z_{2}^{l_{2}} z_{3}^{1-l_{1}-l_{2}}\left|z_{2}\right|^{2 q_{2}}\left|z_{3}\right|^{2 q_{3}}+z_{3}^{l_{2}} z_{2}^{1-l_{1}-l_{2}}\left|z_{3}\right|^{2 q_{2}}\left|z_{2}\right|^{2 q_{3}}\right)
$$




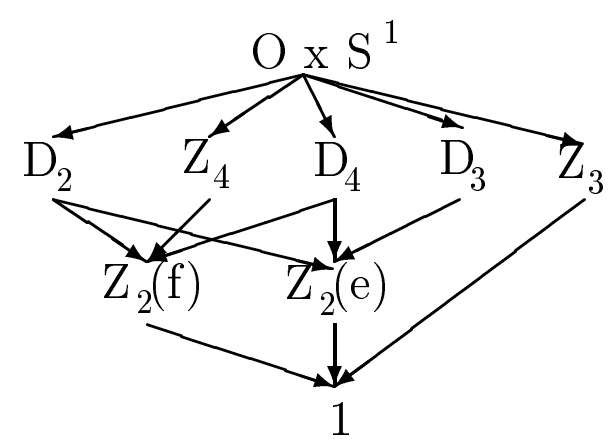

Figure 1: The isotropy lattice for the irreducible action of $\mathbf{O} \times \mathbf{S}^{1}$ on $\mathbf{C}^{3}$.

where $I^{\prime}$ is the subset of $\left(l_{1}, l_{2}, q_{1}, q_{2}, q_{3}\right) \in \mathbf{Z}^{5}$ such that

$$
\left\{\begin{array}{l}
l_{1} \text { odd } \\
l_{2} \geq \frac{1-l_{1}}{2} \text { and } l_{2} \text { even } \\
q_{1} \geq \max \left(-l_{1}, 0\right) \\
q_{2} \geq \max \left(-l_{2}, 0\right) \\
q_{3} \geq \max \left(1-l_{1}-l_{2}, 0\right) .
\end{array}\right.
$$

The other components, $\dot{z}_{2}$ and $\dot{z_{3}}$, can be obtained by cyclic permutation of the indices of $z_{1}, z_{2}$ and $z_{3}$. We show this by considering the formal power series expansion of a vector field on $\mathbf{C}^{3}$ as follows:

$$
\dot{z}_{i}=\sum_{\left(l_{1}, l_{2}, l_{3}\right) \in \mathbf{Z}^{3}} \sum_{\left(q_{1}, q_{2}, q_{3}\right) \in I} C_{l_{1}, l_{2}, l_{3}, q_{1}, q_{2}, q_{3}}^{i} z_{1}^{l_{1}} z_{2}^{l_{2}} z_{3}^{l_{3}}\left|z_{1}\right|^{2 q_{1}}\left|z_{2}\right|^{2 q_{2}}\left|z_{3}\right|^{2 q_{3}}
$$

$(i=1,2,3)$, where

$$
I=\left\{\left(q_{1}, q_{2}, q_{3}\right) \in \mathbf{Z}^{3}: q_{1} \geq \max \left(-l_{1}, 0\right), q_{2} \geq \max \left(-l_{2}, 0\right) \text { and } q_{3} \geq \max \left(-l_{3}, 0\right)\right\} .
$$

The series (6) commutes with the action of $\mathbf{O} \times \mathbf{S}^{1}$ if and only if it commutes with all its generators. It commutes with $\rho_{111}$ if

$$
C_{l_{3}, l_{1}, l_{2}, q_{3}, q_{1}, q_{2}}^{2}=C_{l_{1}, l_{2}, l_{3}, q_{1}, q_{2}, q_{3}}^{1}
$$

and

$$
C_{l_{1}, l_{2}, l_{3}, q_{1}, q_{2}, q_{3}}^{1}=C_{l_{2}, l_{3}, l_{1}, q_{2}, q_{3}, q_{1}}^{3}
$$

for all $l_{i}, q_{i}$. It commutes with $\rho_{001}$ if

$$
\begin{aligned}
& C_{l_{1}, l_{2}, l_{3}, q_{1}, q_{2}, q_{3}}^{1}=(-1)^{l_{2}} C_{l_{2}, l_{1}, l_{3}, q_{2}, q_{1}, q_{3}}^{2}, \\
& C_{l_{1}, l_{2}, l_{3}, q_{1}, q_{2}, q_{3}}^{2}=(-1)^{l_{2}+1} C_{l_{2}, l_{1}, l_{3}, q_{2}, q_{1}, q_{3}}^{1} \text { and } \\
& C_{l_{1}, l_{2}, l_{3}, q_{1}, q_{2}, q_{3}}^{3}=(-1)^{l_{2}} C_{l_{2}, l_{1}, l_{3}, q_{2}, q_{1}, q_{3}}^{3} .
\end{aligned}
$$


Finally, it commutes with $\mathbf{S}^{1}$ if

$$
C_{l_{1}, l_{2}, l_{3}, q_{1}, q_{2}, q_{3}}^{i}=0 \text { for } l_{1}+l_{2}+l_{3} \neq 1
$$

After some algebra the above relations for $C_{\mathbf{1}, \mathbf{q}}^{1}$ can be reduced to $C_{l_{1}, l_{2}, l_{3}, q_{1}, q_{2}, q_{3}}^{1}=0$ if $l_{1}$ is even, $l_{2}$ is odd, or $l_{3}$ is odd and

$$
C_{l_{1}, l_{2}, l_{3}, q_{1}, q_{2}, q_{3}}^{1}=C_{l_{1}, l_{3}, l_{2}, q_{1}, q_{3}, q_{2}}^{1} .
$$

Substitution of the above expressions into (6) for $i=1$ yields the equivariant normal form given.

Alternatively, one can compute that 1 and $\left|z_{1}\right|^{2}+\left|z_{2}\right|^{2}+\left|z_{3}\right|^{2}$ generate the ring of invariants up to cubic orders while the module of equivariants (to cubic order) is generated by

$$
\left(\begin{array}{c}
z_{1} \\
z_{2} \\
z_{3}
\end{array}\right), \quad\left(\begin{array}{c}
\left|z_{1}\right|^{2} z_{1} \\
\left|z_{2}\right|^{2} z_{2} \\
\left|z_{3}\right|^{2} z_{3}
\end{array}\right), \quad\left(\begin{array}{c}
\left(z_{2}^{2}+z_{3}^{2}\right) \overline{z_{1}} \\
\left(z_{1}^{2}+z_{3}^{2}\right) \overline{z_{2}} \\
\left(z_{1}^{2}+z_{2}^{2}\right) \overline{z_{3}}
\end{array}\right)
$$

over the ring of invariants. Hence one can see that the cubic order normal form is given by

$$
\begin{aligned}
& \dot{z_{1}}=(\lambda+i \omega) z_{1}+A_{1}\left|z_{1}\right|^{2} z_{1}+A_{2}\left(\left|z_{2}\right|^{2}+\left|z_{3}\right|^{2}\right) z_{1}+A_{3}\left(z_{2}^{2}+z_{3}^{2}\right) \overline{z_{1}} \\
& \dot{z_{2}}=(\lambda+i \omega) z_{2}+A_{1}\left|z_{2}\right|^{2} z_{2}+A_{2}\left(\left|z_{1}\right|^{2}+\left|z_{3}\right|^{2}\right) z_{2}+A_{3}\left(z_{1}^{2}+z_{3}^{2}\right) \overline{z_{2}} \\
& \dot{z_{3}}=(\lambda+i \omega) z_{3}+A_{1}\left|z_{3}\right|^{2} z_{3}+A_{2}\left(\left|z_{1}\right|^{2}+\left|z_{2}\right|^{2}\right) z_{3}+A_{3}\left(z_{1}^{2}+z_{2}^{2}\right) \overline{z_{3}} .
\end{aligned}
$$

For convenience we write

$$
A_{1}=A_{1 r}+i A_{1 i}, \text { etc. }
$$

We are interested in the case where $\lambda \sim 0$ and $\omega=O(1)$ for Hopf bifurcation. The fifth order normal form has first component given by

$$
\begin{aligned}
\dot{z}_{1} & =(\lambda+i \omega) z_{1}+A_{1}\left|z_{1}\right|^{2} z_{1}+A_{2}\left(\left|z_{2}\right|^{2}+\left|z_{3}\right|^{2}\right) z_{1}+A_{3}\left(z_{2}^{2}+z_{3}^{2}\right) \overline{z_{1}} \\
& +\left(A_{4}\left|z_{1}\right|^{2}\left(z_{2}^{2}+z_{3}^{2}\right)+A_{5}\left(\left|z_{2}\right|^{2} z_{2}^{2}+\left|z_{3}\right|^{2} z_{3}^{2}\right)+A_{6}\left(\left|z_{2}\right|^{2} z_{3}^{2}+\left|z_{3}\right|^{2} z_{2}^{2}\right)\right) \overline{z_{1}} \\
& +\left(A_{7}\left({\overline{z_{2}}}^{2}+{\overline{z_{3}}}^{2}\right) z_{1}^{2}+A_{8}\left|z_{1}\right|^{4}+A_{9}\left(\left|z_{2}\right|^{4}+\left|z_{3}\right|^{4}\right)+A_{10}\left|z_{2} z_{3}\right|^{2}\right) z_{1} .
\end{aligned}
$$

and the other components can be found by cyclic permutation of the indices of $z_{1}, z_{2}$ and $z_{3}$. There can be seen to be seven different equivariants at fifth order. 


\begin{tabular}{cccc} 
Name & Branch & $|x|^{2}$ & No of solutions \\
\hline $\mathbf{D}_{3}$ & $(z, z, z)$ & $-\lambda\left(A_{1 r}+2 A_{2 r}+2 A_{3 r}\right)^{-1}$ & 4 \\
$\mathbf{D}_{2}$ & $(z, z, 0)$ & $-\lambda\left(A_{1 r}+A_{2 r}+A_{3 r}\right)^{-1}$ & 6 \\
$\mathbf{D}_{4}$ & $(z, 0,0)$ & $-\lambda\left(A_{1 r}\right)^{-1}$ & 3 \\
$\mathbf{Z}_{4}$ & $(z, i z, 0)$ & $-\lambda\left(A_{1 r}+A_{2 r}-A_{3 r}\right)^{-1}$ & 6 \\
$\mathbf{Z}_{3}$ & $\left(z, z e^{2 \pi i / 3}, z e^{4 \pi i / 3}\right)$ & $-\lambda\left(A_{1 r}+2 A_{2 r}-A_{3 r}\right)^{-1}$ & 8
\end{tabular}

Table 2: Branching for the maximal symmetry branches at generic Hopf bifurcation with symmetry $\mathbf{O}$. All branches have non-degenerate branching behaviour as determined by the third order normal form.

Maximal symmetry branches All maximal isotropy subgroups for this action of $\mathbf{O} \times$ $\mathbf{S}^{1}$ have fixed point spaces that are one (complex) dimensional; the Equivariant Hopf Lemma [26] then implies that each of these supports a branch of solutions. In this case we can compute the branching behaviour as in Table 2 and their stability in Table 3 . Observe that all branches are different for generic values of the coefficients; this means that branching is not degenerate if we only use the 3rd order normal form. From this information we see that in the case $A_{1 r}<0$ we can get branching of the maximal symmetry solutions in the $\lambda>0$ direction (and hence possible stable branches) for the regions shown in Figure 2. As usual we refer to branches that appear for $\lambda>0$ as supercritical and those for $\lambda<0$ as subcritical. We summarize:

- The branching only depends on $\left(A_{1 r}, A_{2 r}, A_{3 r}\right)$, but the stability depends also on $\left(A_{1 i}, A_{2 i}, A_{3 i}\right)$.

- For the case $A_{1 r}<0$ illustrated, the $\mathbf{D}_{4}$ branch always branches supercritically.

- The case $A_{1 r}>0$ is not illustrated but will lead to a similar pattern of branching depending on $\left(A_{2 r}, A_{3 r}\right)$.

- One can only realize 20 of the 32 possible combinations of sub/supercritical branching of the maximal subgroups by appropriate choice of normal form coefficients.

The stability of the maximal symmetry branches is calculated in Table 3 . The stability is given in terms of eigenvalues $\xi$ for the linearisation about these relative equilibria. These 


\begin{tabular}{c|cccc} 
Name & Type & e.v./ & Branches \\
& & & & to \\
\hline $\mathbf{D}_{3}$ & $\mathrm{r}$ & -2 & 1 & \\
& $\mathrm{t}$ & $\frac{-A_{1 r}+A_{2 r}+4 A_{3 r} \pm \sqrt{\left(A_{1 r}-A_{2 r}+2 A_{3 r}\right)^{2}+12 A_{3 i}\left(A_{1 i}-A_{2 i}-A_{3 i}\right)}}{A_{1 r}+2 A_{2 r}+2 A_{3 r}}$ & 4 & $\mathbf{Z}_{2}(e)$ \\
\hline $\mathbf{D}_{2}$ & $\mathrm{r}$ & -2 & 1 & \\
& $\mathrm{t}$ & $\frac{-A_{1 r}+A_{2 r}+3 A_{3 r} \pm \sqrt{\left(A_{1 r}-A_{2 r}+A_{3 r}\right)^{2}+8 A_{3 i}\left(A_{1 i}-A_{2 i}-A_{3 i}\right)}}{A_{1 r}+A_{2 r}+A_{3 r}}$ & 2 & $\mathbf{Z}_{2}(f)$ \\
& $\mathrm{t}$ & $\frac{A_{1 r}-A_{2 r}+A_{3 r} \pm \sqrt{4\left|A_{3}\right|^{2}-\left(A_{1 i}-A_{2 i}+A_{3 i}\right)^{2}}}{A_{1 r}+A_{2 r}+A_{3 r}}$ & 2 & $\mathbf{Z}_{2}(e)$ \\
\hline $\mathbf{D}_{4}$ & $\mathrm{r}$ & -2 & 1 & \\
& $\mathrm{t}$ & $\frac{A_{1 r}-A_{2 r} \pm \sqrt{\left|A_{3}\right|^{2}-\left(A_{1 i}-A_{2 i}\right)^{2}}}{A_{1 r}}$ & 4 & $\mathbf{Z}_{2}(e, f)$ \\
\hline $\mathbf{Z}_{4}$ & $\mathrm{r}$ & -2 & 2 & \\
& $\mathrm{t}$ & $\frac{A_{1}-A_{2}-A_{3}}{A_{1 r}+A_{2 r}-A_{3 r}, \quad c . c .}$ & 2 & $\mathbf{Z}_{2}(f)$ \\
\hline $\mathbf{Z}_{3}$ & $\mathrm{r}$ & $\frac{-A_{1 r}+A_{2 r}-3 A_{3 r} \pm \sqrt{\left(A_{1 r}-A_{2 r}-A_{3 r}\right)^{2}+8 A_{3 i}\left(-A_{1 i}+A_{2 i}-A_{3 i}\right)}}{A_{1 r}+A_{2 r}-A_{3 r}}$ & 2 & \\
& $\mathrm{t}$ & -2 & 4 & \\
\hline
\end{tabular}

Table 3: Stabilities of the maximal branches of periodic solutions bifurcating at generic Hopf bifurcation with symmetry $\mathbf{O}$. The type refers to whether the eigendirection is $(r)$ radial, ie within the fixed point subspace or (t) transverse out of the fixed point subspace. the column \# refers to the number of eigenvalues with this form. The final column indicates the symmetry types of any periodic solutions that appear at secondary bifurcation associated with these eigenvalues being zero; all branches can also undergo secondary Hopf bifurcation to tori. These stabilities are given in terms eigenvalues of the relative equilibria of the third order normal form coefficients, ignoring the zero eigenvalues along the group orbit of the normal form symmetry $\mathbf{S}^{1}$. The quantities $\xi_{k}$ are roots of the equation (9); these roots come as two complex pairs and c.c. denotes complex conjugate. 
can be converted into Floquet exponents for the periodic orbits (with period $T$ ) simply by computing $e^{\xi T}$.

The eigenvalues of the $\mathbf{Z}_{3}$ solution are given by solving the polynomial equation

$$
\xi^{4}+c_{3} \xi^{3}+c_{2} \xi^{2}+c_{1} \xi+c_{0}=0
$$

where

$$
\begin{aligned}
c_{0}= & 36\left|A_{3}\right|^{2}\left|-A_{1}+A_{2}+A_{3}\right|^{2} \\
c_{1}= & 24\left(\left(-A_{1 r}+A_{2 r}+2 A_{3 r}\right) A_{3 r}^{2}+\left(2 A_{3 i}^{2}-\left(A_{1 r}-A_{2 r}\right)^{2}+A_{3 i}\left(A_{1 i}-A_{2 i}\right)\right) A_{3 r}\right. \\
& \left.+\left(-2 A_{1 r}+2 A_{2 r}\right) A_{3 i}^{2}+\left(A_{1 r}-A_{2 r}\right)\left(-A_{1 i}+A_{2 i}\right) A_{3 i}\right) \\
c_{2}= & 4\left(A_{1 r}^{2}+A_{2 r}^{2}+A_{3 r}^{2}\right)-8 A_{1 r} A_{2 r}+12 A_{3 i}\left(A_{1 i}-A_{2 i}\right) \\
& +24 A_{3 i}^{2}+28 A_{3 r}\left(A_{1 r}-A_{2 r}\right) \\
c_{3}= & -4 A_{1 r}+4 A_{2 r}-8 A_{3 r}
\end{aligned}
$$

We have not been able to simplify this substantially, but this equation appears to have four proper complex roots for almost all parameter values.

The final column in Table 3 giving the symmetry of the submaximal relative equilibria that branch when these eigenvalues pass through zero. These are found by examining the limiting behaviour of the submaximal relative equilibria.

Note that all maximal isotropy subgroups have one complex dimensional fixed point spaces and so are $\mathbf{C}$-axial in terms of [15]. We note that there are 5 such maximal subgroups, and so we can conclude that at such a bifurcation we will have 27 branches of periodic solutions bifurcating with the 5 maximal symmetry types.

\subsection{Dynamics in $\operatorname{Fix}\left(Z_{2}(f)\right)$}

The normal form on $\operatorname{Fix}\left(\mathbf{Z}_{2}(f)\right)$ reduces to the $\mathbf{D}_{4}$ normal form studied by Swift [43], namely if $z_{3}=0$ then

$$
\begin{aligned}
& \dot{z_{1}}=(\lambda+i \omega) z_{1}+A_{1}\left|z_{1}\right|^{2} z_{1}+A_{2}\left|z_{2}\right|^{2} z_{1}+A_{3} z_{2}^{2} \overline{z_{1}} \\
& \dot{z_{2}}=(\lambda+i \omega) z_{2}+A_{1}\left|z_{2}\right|^{2} z_{2}+A_{2}\left|z_{1}\right|^{2} z_{2}+A_{3} z_{1}^{2} \overline{z_{2}}
\end{aligned}
$$

As in [43] one can better understand the dynamics of this system by parametrizing $\mathbf{C}^{2}$ by coordinates $(r, \theta, \phi, \psi) \in \mathbf{R}^{4}$, where

$$
z_{1}=\sqrt{r} \cos \frac{\theta}{2} \exp \left(i \frac{\phi+\psi}{2}\right), z_{2}=\sqrt{r} \sin \frac{\theta}{2} \exp \left(i \frac{-\phi+\psi}{2}\right)
$$




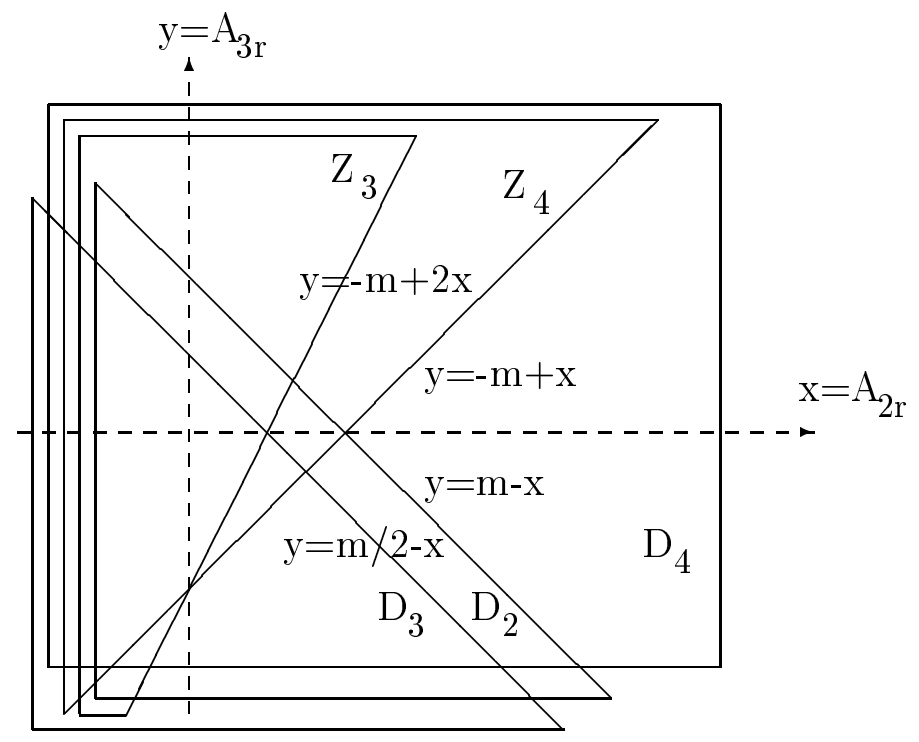

Figure 2: For $A_{1 r}=-m<0$, the maximal symmetry solutions branch in the $\lambda>0$ direction for these values of the normal form coefficients in the $\left(A_{2 r}, A_{3 r}\right)$ plane. The lines where branching changes direction are indicated on this diagram. Observe that there are $\mathbf{D}_{4}$ branches everywhere for $A_{1 r}<0$ and that there are 10 different cases; $A_{1 r}>0$ gives a further 10 cases. 
so that the condition for a periodic orbit $\psi=K t$ can be expressed as

$$
\begin{aligned}
& r \cos \theta=\left|z_{1}\right|^{2}-\left|z_{2}\right|^{2}=K_{1}, \\
& r \sin \theta \exp i \phi=2 z_{1} \bar{z}_{2}=K_{2} .
\end{aligned}
$$

where $K_{1} \in \mathbf{R}$ and $K_{2} \in \mathbf{C}$ are constants. Observe that the $\mathbf{S}^{1}$ coordinate $\psi$ is removed by this change of coordinates. Hence we can find the locations of periodic orbits in (7) by differentiating the above expressions to obtain the equations

$$
\begin{aligned}
r\left(2 \cos \theta\left(A_{1 r} r+\lambda\right)+\left(r A_{3 i} \sin ^{2} \theta\right) \sin 2 \theta\right) & =0 \\
r \sin \theta\left(\lambda+r\left(\frac{1}{2} A_{1 r}+\frac{1}{2} A_{2 r}+A_{3 r}\left(-\frac{1}{2}+\cos ^{2} \phi\right)-A_{3 i} \cos \theta \sin \phi \cos \phi\right)\right) & =0 \\
r^{2} \sin \theta\left(\cos \theta\left(\frac{1}{2}\left(A_{1 i}-A_{2 i}+A_{3 i}\right)-A_{3 i} \cos ^{2} \phi\right)-A_{3 r} \sin \phi \cos \phi\right) & =0 .
\end{aligned}
$$

Note that from these equations we can recover (a) the trivial solution $r=0$, (b) the maximal branches where $\sin \theta=0$ or $\cos \theta=0$, (c) the submaximal branches where $\theta$ and $\phi$ vary depending on the normal form coefficients.

Submaximal branches in $\mathbf{F i x}\left(\mathbf{Z}_{2}(f)\right)$ Restricting by excluding the cases (a-c) above we can obtain submaximal branches as follows: solving (13) we get

$$
\sin 2 \phi=\frac{2 \cos \theta\left(r A_{1 r}+\lambda\right)}{r A_{3 i} \sin ^{2} \theta}
$$

whereas solving (15) we obtain

$$
\cos \theta=\frac{A_{3 r} \sin 2 \phi}{A_{2 i}-A_{1 i}+A_{3 i} \cos 2 \phi}
$$

Moreover rearranging (14) we get

$$
r=\frac{-2 \lambda}{A_{1 r}+A_{2 r}+A_{3 r} \cos 2 \phi+A_{3 i} \cos \theta \sin 2 \phi}
$$

and using this to eliminate $r$, we can define $P_{4}$ and $P_{5}$ by

$$
P_{4}:=\cos 2 \phi=\frac{A_{3 r}\left(A_{1 r}-A_{2 r}\right)+A_{3 i}\left(A_{1 i}-A_{2 i}\right)}{A_{3 r}^{2}+A_{3 i}^{2}}=\frac{\operatorname{Re}\left(\bar{A}_{3}\left(A_{1}-A_{2}\right)\right)}{\left|A_{3}\right|^{2}}
$$

and

$$
P_{5}:=\cos ^{2} \theta=\frac{\left|A_{3}\right|^{4}-\left|\operatorname{Re}\left(\bar{A}_{3}\left(A_{1}-A_{2}\right)\right)\right|^{2}}{\left|\operatorname{Im}\left(\bar{A}_{3}\left(A_{1}-A_{2}\right)\right)\right|^{2}} .
$$

Hence there will be a branch of submaximals with symmetry $Z_{2}(f)$ if and only if

$$
-1<P_{4}<1 \quad \text { and } 0<P_{5}<1
$$


where $P_{4}$ and $P_{5}$ are real functions of the cubic order coefficients as defined above. The direction of branching will depend of the sign of the denominator of (18). Note that these expressions are equivalent to the slightly more compact expressions of Swift [43], but they are expressed in the original variables rather than transformed ones.

On varying one of the normal form coefficients as a second parameter, the $\mathbf{Z}_{2}(f)$ submaximal branches will limit to the following maximal isotropy subspaces as follows:

\begin{tabular}{l|llll} 
Limit & $\left(z_{1}, z_{2}\right)$ & $(\theta, \phi)$ & $\left(P_{4}, P_{5}\right)$ & Branching \\
\hline $\mathbf{D}_{4}$ & $z_{2}=0$ & $\theta=0$ & $P_{5}=1$ & $\left|A_{3}\right|=\left|A_{1}-A_{2}\right|$ \\
$\mathbf{D}_{2}$ & $z_{1}=z_{2}$ & $\theta=\pi / 2, \phi=0, \pi$ & $P_{4}=1, P_{5}=0$ & $\left|A_{3}\right|^{2}=\operatorname{Re}\left(\bar{A}_{3}\left(A_{1}-A_{2}\right)\right)$ \\
$\mathbf{Z}_{4}$ & $z_{2}=i z_{1}$ & $\theta=\pi / 2, \phi=\pi / 2$ & $P_{4}=-1, P_{5}=0$ & $\left|A_{3}\right|^{2}=\operatorname{Re}\left(\bar{A}_{3}\left(A_{2}-A_{1}\right)\right)$
\end{tabular}

The conditions for branching from maximals to $\mathbf{Z}_{2}(f)$ are found from the expressions for $P_{4}$ and $P_{5}$ and it can be checked that these give rise to a zero eigenvalue for the relevant maximal solution.

\subsection{Dynamics in $\operatorname{Fix}\left(\mathrm{Z}_{2}(e)\right)$}

On the invariant subspace $\left(z_{1}, z_{2}, z_{2}\right)$ the dynamics is given by

$$
\begin{aligned}
& \dot{z_{1}}=(\lambda+i \omega) z_{1}+A_{1}\left|z_{1}\right|^{2} z_{1}+2 A_{2}\left|z_{2}\right|^{2} z_{1}+2 A_{3} z_{2}^{2} \overline{z_{1}} \\
& \dot{z_{2}}=(\lambda+i \omega) z_{2}+\left(A_{1}+A_{2}+A_{3}\right)\left|z_{2}\right|^{2} z_{2}+A_{2}\left|z_{1}\right|^{2} z_{2}+A_{3} z_{1}^{2} \overline{z_{2}} .
\end{aligned}
$$

One can perform the same substitution (11) to obtain submaximal branches when

$$
\begin{aligned}
\lambda+\frac{r}{2}\left[A_{1 r}+2 A_{2 r}-A_{3 r}+(3 \cos \theta-1)\left(A_{3 r} \cos ^{2} \phi+A_{3 i} \cos \phi \sin \phi\right)\right] & =0 \\
\frac{1}{2} \cos \theta\left(A_{1 i}-A_{2 i}+2 A_{3 i}+A_{3 r} \sin \phi \cos \phi-A_{3 i} 3 \cos ^{2} \phi\right) & \\
+\frac{A_{3 i}}{2}\left(\cos ^{2} \phi-1\right)-\frac{3}{2} A_{3 r} \sin \phi \cos \phi & =0 \\
r A_{3 r}\left(\cos ^{2} \phi-1\right)+3 r A_{3 i} \sin \phi \cos \phi+\cos \theta\left(2 \lambda+r\left(2 A_{1 r}+A_{2 r}-A_{3 r}\right)\right) & \\
+r \cos ^{2} \theta\left(-A_{2 r}+A_{3 r} \cos ^{2} \phi-3 A_{3 i} \sin \phi \cos \phi\right) & =0
\end{aligned}
$$

The above equations do not have the symmetries of the $\mathbf{Z}_{2}(f)$ equations with the consequence that we have not been able to solve them explicitly. Nevertheless, their branching behaviour is computable.

Submaximal branches in $\operatorname{Fix}\left(\mathbf{Z}_{2}(e)\right)$ In this subspace there can be branches of periodic solutions with submaximal symmetry, namely when (21-23) have nontrivial solutions $(r, \theta, \phi)$. 
These solutions limit onto the $\mathbf{D}_{2}$ maximal branch when $\cos \theta=0$ and onto the $\mathbf{D}_{4}$ maximal branch when $\cos \theta=-1$. They bifurcate from the $\mathbf{D}_{3}$ maximal branch when $\sin \theta=\cos \theta$ and $\phi=0$. In other words, these solutions branch from maximal solutions under the following conditions

\begin{tabular}{l|lll} 
Limit & $\left(z_{1}, z_{2}\right)$ & $(\theta, \phi)$ & Branching \\
\hline $\mathbf{D}_{2}$ & $z_{1}=0$ & $\theta=\pi$, & $2\left|A_{3}\right|=\left|A_{1}-A_{2}+A_{3}\right|$ \\
$\mathbf{D}_{4}$ & $z_{2}=0$ & $\theta=0$, & $\left|A_{3}\right|=\left|A_{2}-A_{1}\right|$ \\
$\mathbf{D}_{3}$ & $z_{1}=z_{2}$ & $\theta=\pi / 2, \phi=0$ & $\left|A_{3}\right|^{2}=\operatorname{Re}\left(\bar{A}_{3}\left(A_{1}-A_{2}\right)\right)$
\end{tabular}

Note that there can be saddle-node bifurcations within $\operatorname{Fix}\left(\mathbf{Z}_{2}(e)\right)$ when $\sin ^{2} \phi=1, r \neq 1$ and $\theta \neq 0$. These are not associated with bifurcation to or from any invariant subspace.

\section{Examples of attracting behaviour}

We now attempt to understand the possible dynamics of the normal form (7). Note that even for the $\mathbf{D}_{4}$ Hopf bifurcation [43] proving the exact form and genericity of the branch of quasiperiodic solutions is impossible if one includes normal form symmetry breaking terms, due to the appearance of resonances. In this section we use the dynamical systems package dstool_tk [28] with the 'quality controlled' 4th order Runge-Kutta method.

\subsection{Symmetries of attractors}

We say a compact invariant set $A$ is an attractor, if it is the $\omega$-limit set of a positive measure set of initial conditions (for example, see the discussion in [5]). It is important to make a distinction between two kinds of symmetries that can be attributed to an attractor in a symmetric system. Given an attractor $A$, we define $\mathbf{T}(A)=\{\sigma \in G: \sigma(x)=x$ for all $x \in$ $A\}$ and $\Sigma(A)=\{\sigma \in G: \sigma(A)=A\}$ as in [13]. The former subgroup we refer to as the point symmetry of the attractor whereas the latter subgroup is the symmetry on average of the attractor. In this paper we employ the latter notion to discuss the symmetry of an attractor; the former notion corresponds to the isotropy type of a typical point on the attractor which may have less symmetry than the attractor.

Projection onto $\mathbf{S}^{1}$-orbits The $\mathbf{S}^{1}$ symmetry of the normal form means that periodic orbits can be reduced to equilibria on factoring out this symmetry. This allows us to locate 


\begin{tabular}{l|cccc} 
Case & $\lambda$ & $A_{1}$ & $A_{2}$ & $A_{3}$ \\
\hline $\mathrm{I}_{\kappa}$ & $1+i$ & $-1-0.9 i$ & $-0.2+0.45 i$ & $-0.3+\kappa i$ \\
$\mathrm{II}_{\kappa}$ & $1+i$ & $-1-0.9 i$ & $-0.1+0.1 i$ & $-0.3+\kappa i$
\end{tabular}

Table 4: The two families of parameter values of the coefficients in (7) investigated numerically in Section 3.2.

and continue branches of periodic (resp. quasiperiodic) orbits as if they were equilibria (resp. periodic orbits). To this end we project the 3 rd order normal form (7) onto an $\mathbf{R}$-codimension one section in $\mathbf{C}^{3}$ as follows. We define an ODE on $\left(v_{1}, v_{2}, v_{3}\right) \in \mathbf{C}^{3}$ by

$$
\begin{aligned}
& \dot{v}_{1}=f_{1}\left(v_{1}, v_{2}, v_{3}\right)+i a v_{1} \\
& \dot{v}_{2}=f_{2}\left(v_{1}, v_{2}, v_{3}\right)+i a v_{2} \\
& \dot{v}_{3}=f_{3}\left(v_{1}, v_{2}, v_{3}\right)+i a v_{3}
\end{aligned}
$$

where the original equations (7) are expressed as $\dot{z}_{i}=f_{i}\left(z_{1}, z_{2}, z_{3}\right)$, and where we choose

$$
a=\frac{-\operatorname{Im}\left(f_{1} \bar{z}_{1}\right)}{\left|z_{1}\right|^{2}}
$$

This equation is well-defined for all $z_{1} \neq 0$ and its solutions are in correspondence with group orbits of those of (7) in the sense that

$$
v_{k}(t)=z_{k}(t) \exp (i \gamma(t))
$$

for $\gamma(t)$ such that for all $\phi \in[0,2 \pi)$ the spaces

$$
S_{\phi}=\left\{\left(v_{1}, v_{2}, v_{3}\right):\left|v_{1}\right|>0 \text { and } \arg \left(v_{1}\right)=\phi\right\}
$$

are invariant; this enables us to study periodic orbits of (7) by studying equilibria of (24). By choosing initial conditions with $x_{1}$ real and positive, we will remain in the subspace $S_{0}$ for all time and hence effectively reduce the dimension of the system from six to five.

There are bifurcations apparent between chaotic attractors in the cubic normal form system (7) at some example parameter values. These transitions appear to be typical in this system, although proving genericity is only possible in a very limited sense. Table 4 gives the values of the normal form parameters that we investigate here.

\subsection{Numerical examples}

Bifurcations in the family $\mathbf{I}_{\kappa}$ The family of systems $I_{\kappa}$ (parametrized by $\kappa$ ) in Table 4 can be observed to have a number of attractors and bifurcations that start to show the 
On separate page

Figure 3: Bifurcation diagrams showing details of some of the bifurcations for the family $I_{\kappa}$, computed using xppaut. (a) shows the bifurcations on varying $\kappa$ against $\operatorname{Re}\left(x_{3}\right)$ while (b) shows it against $\operatorname{Im}\left(x_{3}\right)$. The diagrams (c) and (d) show the same diagram over a smaller range of $\kappa$. All lines represent relative equilibria and the horizontal lines represent relative equilibria with maximal symmetry; the thick lines are stable. Note that the only relative equilibria that are stable are the $\mathbf{Z}_{3}$ solutions for $\kappa<0.025$. The circles are branches of stable periodic solutions that branch at a Hopf bifurcation at A. This branch is destroyed at a homoclinic bifurcation at $B . C, D$ and $E$ are bifurcations of submaximal branches with symmetry $\mathbf{Z}_{2}(e)$ and $\mathbf{Z}_{2}(f)$ from the maximal branches. Observe that there are two folds of the branch of $\mathbf{Z}_{2}(e)$ solutions between $B$ and $C$ on this diagram. The values of $\kappa$ at $A, B, C, D, E$ can be found numerically using the tables in Section 2 and are 0.02514 , $0.04465,0.10322,0.32564$ and 0.59563 respectively.

dynamical richness of the normal form (7). Figure 3, computed using xppaut [17] shows some branches of relative equilibria and periodic orbits in the family for the 'interesting' range of $-0.2<\kappa<0.7$. The solutions are shown projected into the $\operatorname{Re}\left(x_{3}\right)$ and $\operatorname{Im}\left(x_{3}\right)$ components for the $\mathbf{S}^{1}$ reduced system (24) with $\operatorname{Re}\left(x_{1}\right)>0$ and $\operatorname{Im}\left(x_{1}\right)=0$, where $x_{i}=\operatorname{Re}\left(v_{i}\right)$. The steady states and periodic orbits of the reduced system correspond to periodic orbits and tori of the original system (7), respectively. Note that varying $\kappa$ does not change the position of the maximal solutions, only their stability. Hence they appear as horizontal lines on these diagrams. The figure shows only relative equilibria and the $\mathbf{Z}_{3}$ periodic orbits. For $\kappa>0.045$ all of the solutions on this digram are unstable; however they are all of saddle type and many appear to be embedded within the chaotic attractor for larger $\kappa$.

Figure 4 shows attractors for the family $I_{\kappa}$. There is a transition from stable $\mathbf{Z}_{3}$ periodic orbit to fully symmetric chaos; A Poincaré section of the case (d) is shown in Figure 5(a) while (b) shows the network of heteroclinic connections that occur at the isolated parameter value in $I_{\kappa}$ associated with a transition from $\mathbf{Z}_{3}$ tori to $\mathbf{Z}_{4}$ tori. Figure 6 shows the transition at $I_{0.0047}$ schematically in a Poincaré section; before the bifurcation there are $\mathbf{Z}_{3}$ symmetric tori which become $\mathbf{Z}_{4}$ symmetric after the bifurcation. 
(a)

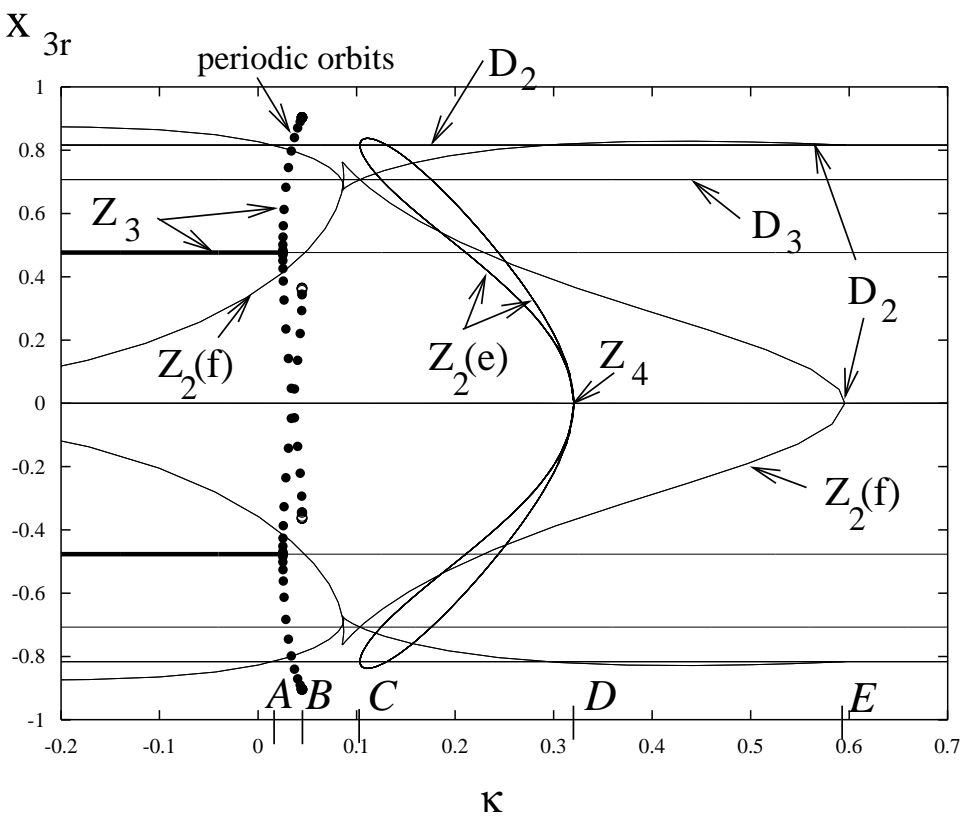

(b)

$x_{3 i}$

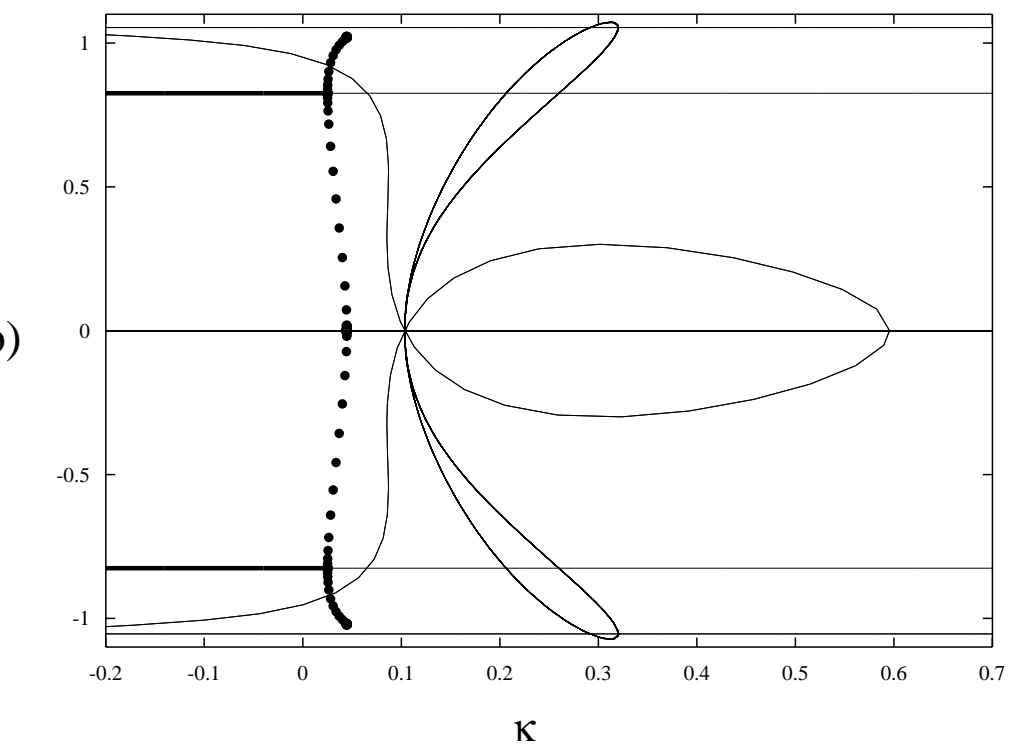

(c)

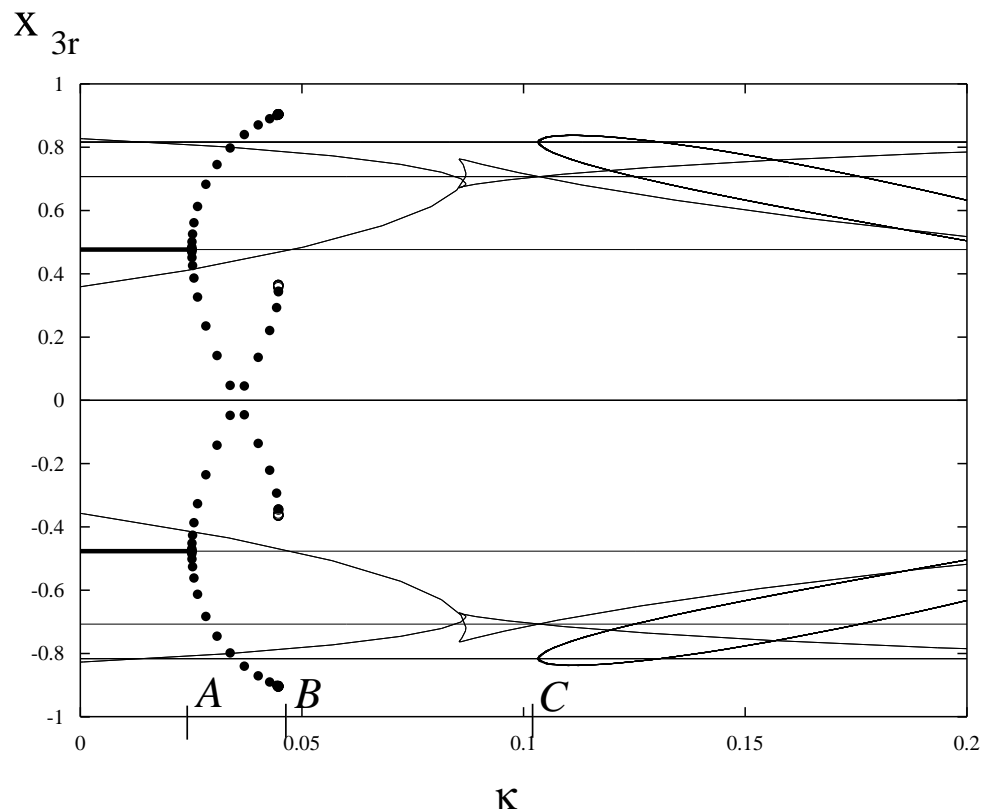

$\mathrm{X}_{3 \mathrm{i}}$

(d)

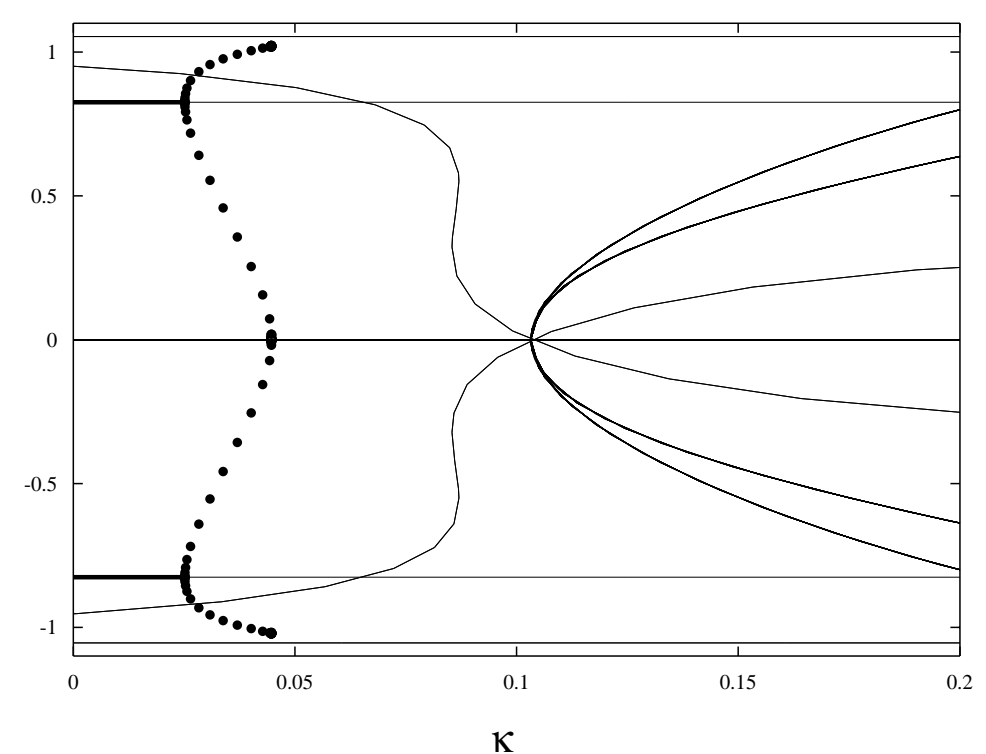

จิ

ڤొ 


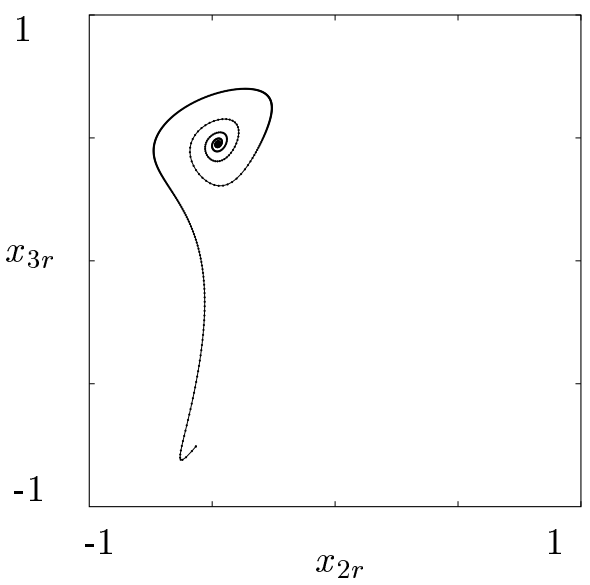

(a)

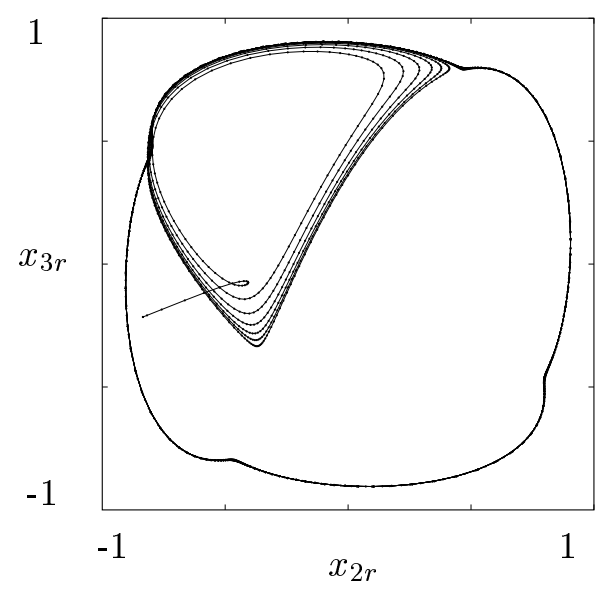

(c)

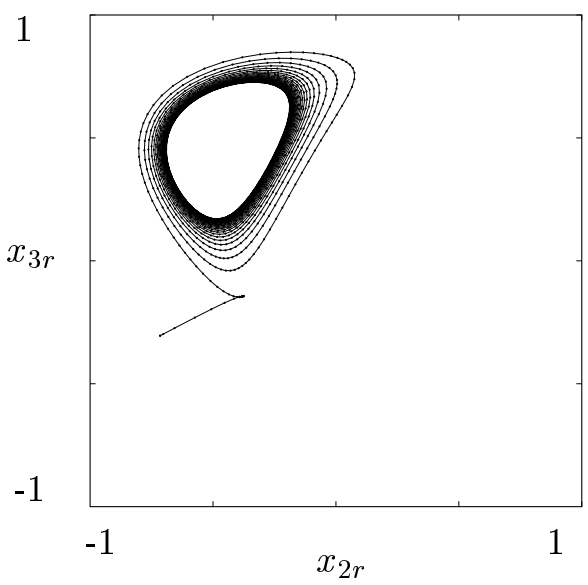

(b)

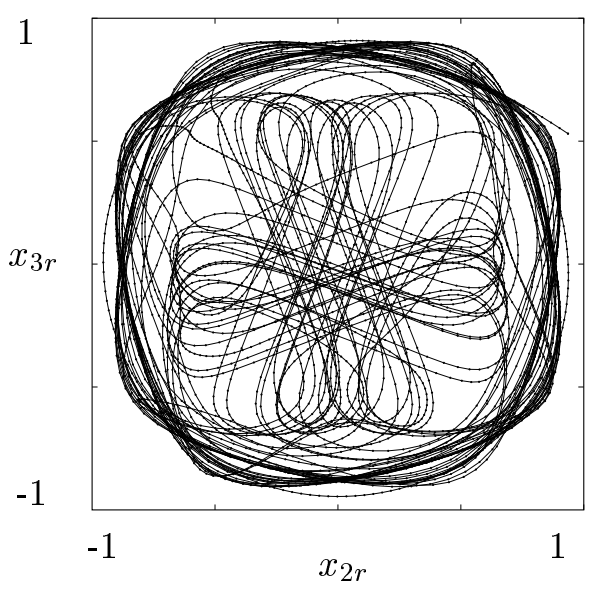

(d)

Figure 4: Attractors for the system (7) are shown with transient behaviour for parameter values (a) $I_{-0.05}$, a stable $\mathbf{Z}_{3}$ relative equilibrium, (b) $I_{0.03}$, a stable $\mathbf{Z}_{3}$ relative periodic orbit, (c) $I_{0.045}$, a stable $\mathbf{Z}_{4}$ relative periodic orbit, (d) $I_{0.2}$, a fully symmetric attractor formed by merging of all $\mathbf{Z}_{4}$ relative periodic orbits. In all cases, the attractors are shown in the $\left(x_{2 r}, x_{3 r}\right)$ plane for the system (24) with the continuous symmetry projected out. In these figures, the projection (and section) preserves a $\mathbf{D}_{4}$ symmetry that fixes $x_{1} i=0$. 


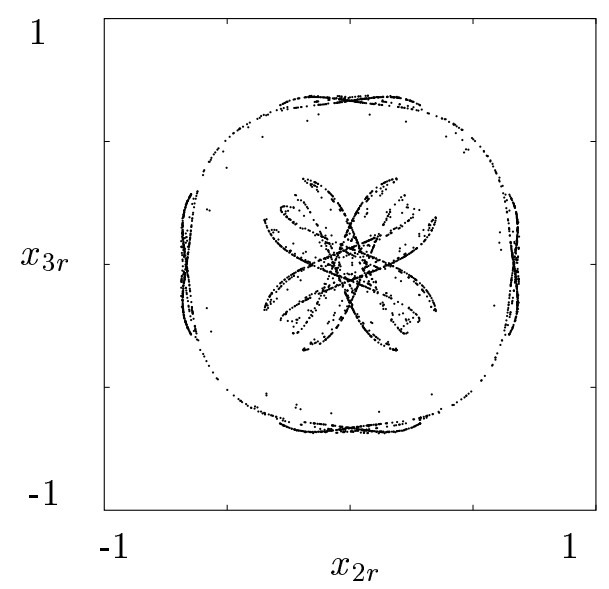

(a)

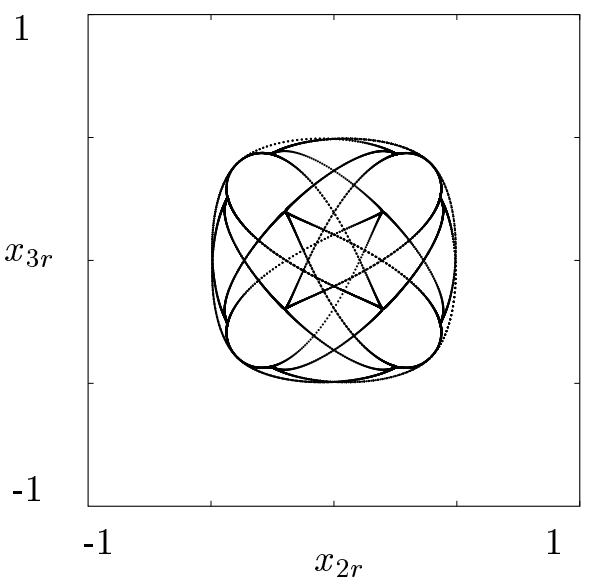

(b)

Figure 5: (a) is a Poincaré section taken at $\operatorname{Re}\left(x_{1}\right)=0.96$ for $I_{0.2}$. (b) $I_{0.0047}$; two equilibria of type $\mathbf{Z}_{2}(e)$ and their unstable manifolds are shown; these are close to forming a network of heteroclinic connections.

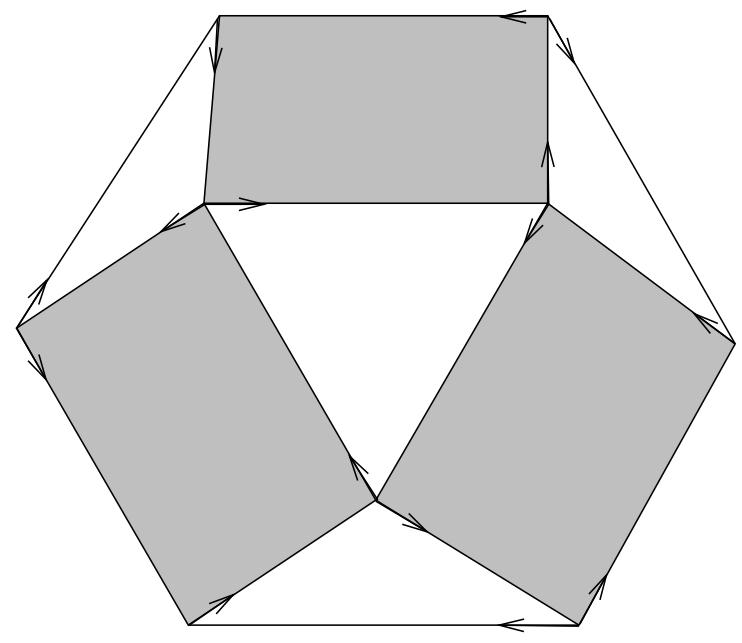

Figure 6: Schematic of a heteroclinic network between periodic orbits with symmetry $\mathbf{Z}_{2}(e)$ (i.e. $\left.\left(z_{1}, z_{1}, z_{2}\right)\right)$. This is formed at the bifurcation where $\mathbf{Z}_{3}$ symmetric tori are replaced by $\mathbf{Z}_{4}$ symmetric tori shown with the $\mathbf{S}^{1}$ symmetry factored out and so the periodic orbits are replaced by equilibria. Before the bifurcation the connections are broken such that there are periodic orbits on the triangular faces corresponding to $\mathbf{Z}_{3}$ tori in the full system. After the bifurcation there are $\mathbf{Z}_{4}$ symmetric relative periodic orbits on the shaded square faces. 
The family $\mathbf{I I}_{\kappa}$ On increasing $\kappa$ this has a branch of stable $\mathbf{Z}_{3}$ periodic orbits that have a subcritical bifurcation leading directly to fully symmetric chaos.

\subsection{Breaking the normal form symmetry}

As previously stated, the $\mathbf{S}^{1}$ normal form symmetry is not present in the full dynamics. Although it does not affect the branching of periodic attractors and their stability, it will cause degeneracies of any more complicated attractors. In particular, tori will only be visible in the dynamics of the full system for isolated parameter values as the presence of resonances will cause breakup of tori at what may be a very small scale.

To examine the effects of normal form symmetry breaking we simulate the system

$$
\begin{aligned}
& \dot{z}_{1}=f_{1}\left(z_{1}, z_{2}, z_{3}\right)+\epsilon z_{1}^{3} \\
& \dot{z}_{2}=f_{2}\left(z_{1}, z_{2}, z_{3}\right)+\epsilon z_{2}^{3} \\
& \dot{z}_{3}=f_{3}\left(z_{1}, z_{2}, z_{3}\right)+\epsilon z_{3}^{3}
\end{aligned}
$$

with $f_{i}$ the cubic order normal form in (7). The extra term $\epsilon z_{i}^{3}$ breaks the $\mathbf{S}^{1}$ symmetry for $\epsilon \neq 0$ while retaining the $\mathbf{O}$ symmetry. Figure 7 (a) and (b) show one of the conjugate attractors for the parameters $\mathrm{I}_{0.028}$ with $\epsilon=-0.047$; the detail (b) shows the presence of small scale folding and presumably chaos in the attractor. (c) Shows an attractor for $\mathrm{I}_{0.035}$ with $\epsilon=-0.047$ that is apparently chaotic and possesses full symmetry. This perturbation can cause the branch of $\mathbf{Z}_{3}$-symmetric tori to break up into a chaotic attractor before merging into a single fully symmetric chaotic attractor. Note that the observed quantitative effect of the symmetry breaking terms on the fully symmetric attractor is not great.

\section{Instability of ABC flow.}

In this section we consider time-dependent solutions to the Navier-Stokes equation with the 1:1:1 ABC forcing. We study in detail the bifurcations, occurring immediately after the trivial steady state - the 1:1:1 ABC flow $(1,2)$ - becomes unstable in a Hopf bifurcation at $R \approx 13.044$. According to [38], the action $\mathcal{H}$ of the group on the center eigenspace is isomorphic to the representation $W_{0}$ (see Section 1), and hence results of Section 2 can be applied to this bifurcation. 


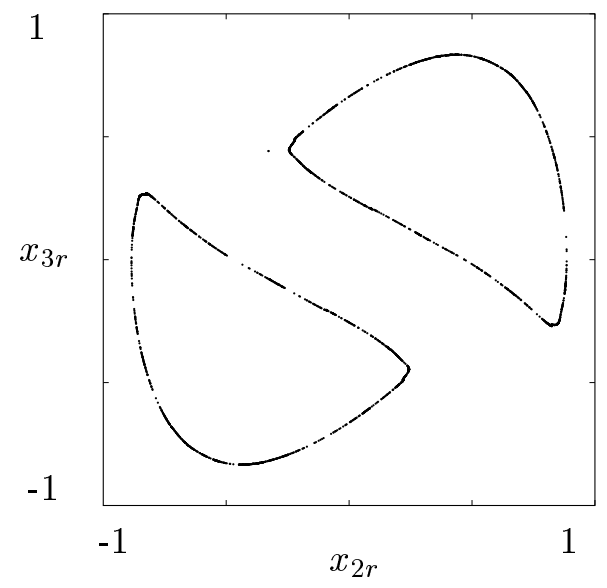

(a)

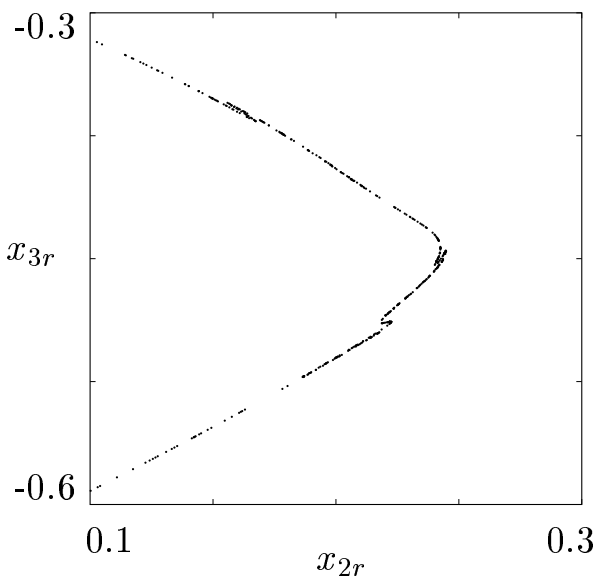

(b)

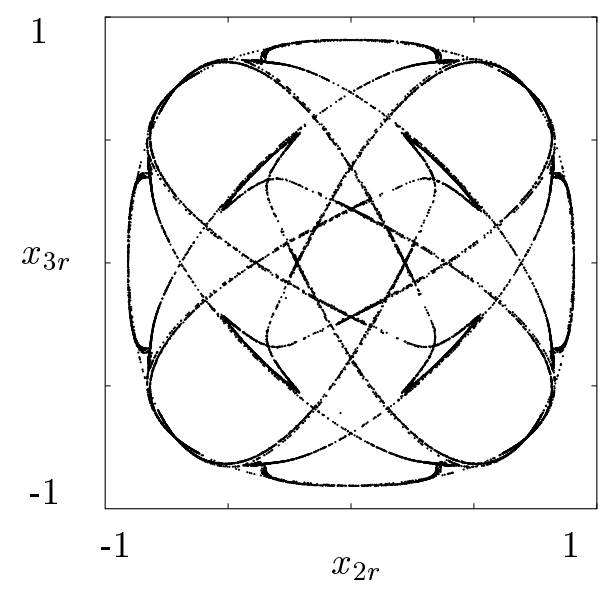

(c)

Figure 7: Simulations of the normal form with broken $\mathbf{S}^{1}$ symmetry, (26) with $x_{i}=\operatorname{Re}\left(z_{i}\right)$, a Poincaré section taken at $x_{1}$ constant. (a) and (b) show a conjugate attractor for $I_{0.028}$, $\epsilon=-0.047 ;$ (c) shows an attractor for $I_{0.035}$ with $\epsilon=-0.047$ with fully symmetric chaos. 


\subsection{The symmetry group of the Navier-Stokes equation with the 1:1:1 $\mathrm{ABC}$ force.}

The symmetry group $\mathcal{H}$ of $(3,4)$ with forcing $(1,2,5)$ is generated by

$$
s_{1}: x_{1} \rightarrow x_{2}, x_{2} \rightarrow x_{3}, x_{3} \rightarrow x_{1}
$$

and

$$
s_{2}: x_{1} \rightarrow \frac{\pi}{2}-x_{2}, x_{2} \rightarrow \frac{\pi}{2}+x_{1}, x_{3} \rightarrow-\frac{\pi}{2}+x_{3} .
$$

Let $\vartheta: \mathbf{O} \rightarrow \mathcal{H}$ be the isomorphism such that $\vartheta\left(\rho_{111}\right)=s_{1}$ and $\vartheta\left(\rho_{001}\right)=s_{2}$. Some other elements of the group are

$$
\vartheta\left(\kappa_{110}^{+}\right)=s_{3}: x_{1} \rightarrow \frac{\pi}{2}+x_{2}, x_{2} \rightarrow-\frac{\pi}{2}+x_{1}, x_{3} \rightarrow \frac{\pi}{2}-x_{3}
$$

and

$$
\vartheta\left(\kappa_{110}^{-}\right)=s_{4}: x_{1} \rightarrow-\frac{\pi}{2}-x_{2}, x_{2} \rightarrow-\frac{\pi}{2}-x_{1}, x_{3} \rightarrow-\frac{\pi}{2}-x_{3} .
$$

\subsection{Numerical simulations.}

Standard pseudospectral methods are used for numerical solution of (3-5). The flow is represented as a Fourier series:

$$
\mathbf{v}(t)=\sum_{\mathbf{k}} \mathbf{v}_{\mathbf{k}}(t) e^{i \mathbf{k} \cdot \mathbf{x}} .
$$

The resolution provided by $16^{3}$ Fourier harmonics suffices and is used throughout. We have reproduced results of some $16^{3}$ harmonics computations, using the resolution of $24^{3}$ harmonics; see the next section for details.

For the action of $\mathcal{H} \cong \mathbf{O}$, the space of $2 \pi$-periodic functions can be decomposed into five isotypic components, corresponding to five representations of the group $\mathbf{O}$. These functional subspaces were described in [38] by relations between Fourier coefficients of their elements. In particular, the quantities

$$
\begin{gathered}
q_{1}(\mathbf{v})=0.5\left(\operatorname{Re}\left(v_{100}^{2}\right)+\operatorname{Im}\left(v_{100}^{3}\right)\right), \quad q_{2}(\mathbf{v})=0.5\left(\operatorname{Re}\left(v_{010}^{3}\right)+\operatorname{Im}\left(v_{010}^{1}\right)\right) \\
q_{3}(\mathbf{v})=0.5\left(\operatorname{Re}\left(v_{001}^{1}\right)+\operatorname{Im}\left(v_{001}^{2}\right)\right)
\end{gathered}
$$

vanish for functions from any of the four subspaces not associated with the representation $W_{0}$. It is convenient to use the $q_{i}$ 's to describe symmetries of attractors, because a symmetry $s_{i} \in \mathcal{H}$ transforms these three quantities in the same way as $\vartheta^{-1}\left(s_{i}\right) \in \mathbf{O}$ transforms the three coordinates of a vector. 


\subsection{Bifurcations to $\mathrm{Z}_{3}$ symmetric attractors}

Beyond the Hopf bifurcation, for $R>R_{0}$ we observe appearance of eight symmetrically related attracting periodic orbits with symmetry $\mathbf{Z}_{3}$ (see Table 1). Plots of the quantities $q_{i}(\mathbf{v}), i=1-3$ (shown on Figure 8 a for one of the orbits for $R=13.05$ ) are shifted by one third of the period. The third order symmetry $s_{1}=\vartheta\left(\rho_{111}\right)$ permutes the values of $q_{i}$ 's:

$$
q_{1}(\mathbf{u})=q_{2}(\mathbf{v}), \quad q_{2}(\mathbf{u})=q_{3}(\mathbf{v}), \quad q_{3}(\mathbf{u})=q_{1}(\mathbf{v}),
$$

where $\mathbf{u}=s_{1}(\mathbf{v})$. Other symmetries from the group $\mathcal{H}$ permute the values and may also change signs, e.g. for $\mathbf{u}=s_{2}(\mathbf{v})$

$$
q_{1}(\mathbf{u})=q_{2}(\mathbf{v}), \quad q_{2}(\mathbf{u})=-q_{1}(\mathbf{v}), \quad q_{3}(\mathbf{u})=q_{3}(\mathbf{v})
$$

and for $\mathbf{u}=s_{3}(\mathbf{v})$

$$
q_{1}(\mathbf{u})=q_{2}(\mathbf{v}), \quad q_{2}(\mathbf{u})=q_{1}(\mathbf{v}), \quad q_{3}(\mathbf{u})=-q_{3}(\mathbf{v}) .
$$

Application of symmetries from $\mathcal{H}$ yields eight symmetric attractors with all eight possible combinations of signs of time averages of the three quantities (note that time averages of $q_{i}$ for any $i$ do not vanish). Thus signs of the time averages of $q_{i}$ label the eight attractors (for example, see time series for the $(+++)$ orbit on Figure 8a). Projection of the trajectory for $R=13.05$ in the saturated regime into the complex plane $q_{1}(\mathbf{v})+\exp (2 \pi i / 3) q_{2}(\mathbf{v})+$ $\exp (-2 \pi i / 3) q_{3}(\mathbf{v})$ is a circle (see Figure $9 \mathrm{a}$ ), indicating that the trajectory possesses the symmetry $S_{1}$. For $13.06 \leq R \leq 13.09$ the solution is also periodic.

At the interval $13.05 \leq R \leq 13.13$ the system has eight symmetry related attractors. Bifurcations of each of them are apparently not affected by existence of its seven symmetric counterparts at this interval of the Reynolds number. Bifurcations of only one of the eight attractors will be described in what follows, the other seven evidently undergoing the same bifurcations.

For $R=13.1$ the behaviour is quasi-periodic: a torus with the second frequency, $f_{2} \approx 0.0017$, much smaller than the first one, $f_{1} \approx 0.043$ (cf. Figures 11a,b) emerges in a Hopf bifurcation from the periodic orbit at $R=R_{1}\left(13.09<R_{1}<13.1\right)$. The second frequency is visible in the time evolution of $q_{1}(\mathbf{v})$ for $R=13.1$ (see Figure $8 \mathrm{~b}$ ). Figure $9 \mathrm{~b}$, showing projection of the trajectory in the saturated regime into the complex plane $q_{1}(\mathbf{v})+\exp (2 \pi / 3 i) q_{2}(\mathbf{v})+\exp (-2 \pi / 3 i) q_{3}(\mathbf{v})$, demonstrates that the attractor lacks 


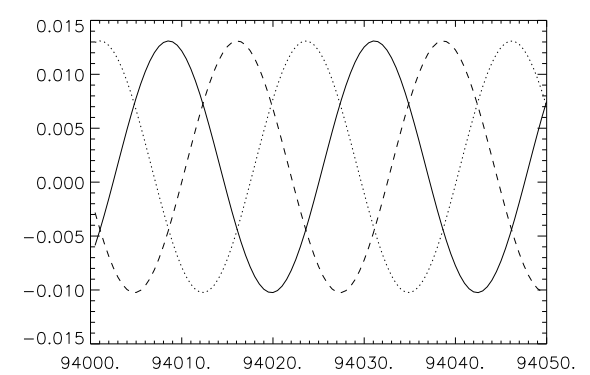

(a)

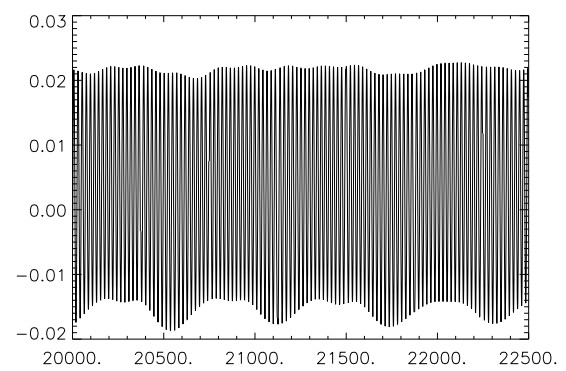

(c)

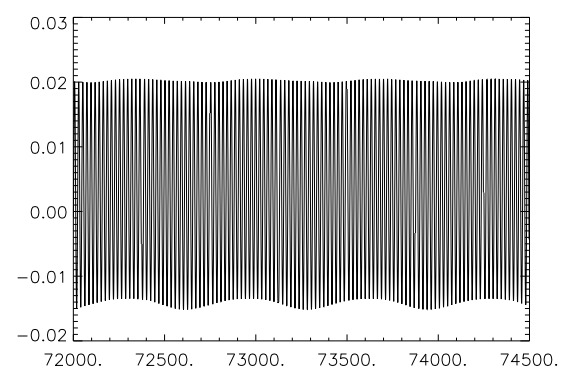

(b)

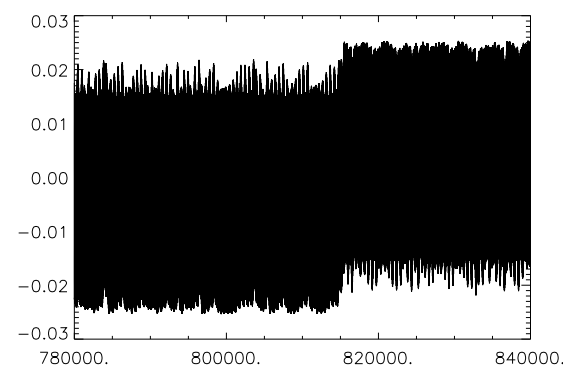

(d)

Figure 8: The quantities $q_{i}(\mathbf{v}(t)), i=1-3$ (a) or $i=1$ (b-d) (vertical axis) versus time (horizontal axis) for $R=13.05$ (a), $R=13.1$ (b), $R=13.12$ (c) and $R=13.14$ (d).

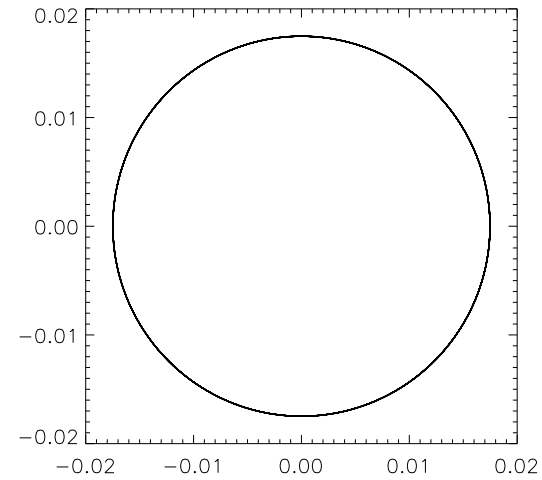

(a)

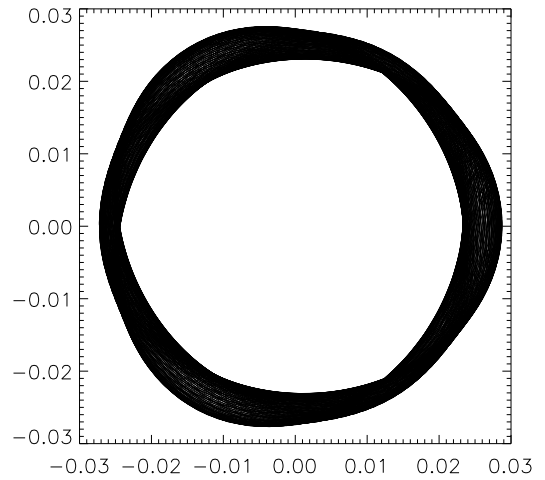

(b)

Figure 9: Projection of the trajectory in the saturated regime into the complex plane $q_{1}(\mathbf{v}(t))+\exp (2 \pi i / 3) q_{2}(\mathbf{v}(t))+\exp (-2 \pi i / 3) q_{3}(\mathbf{v}(t))$ for $R=13.05$ (a) and $R=13.1$ (b). 


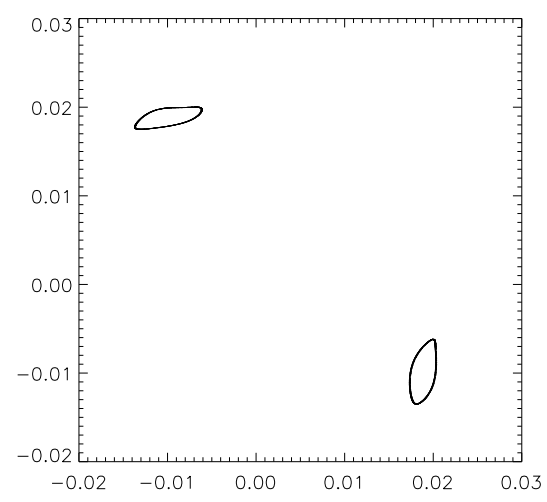

(a)

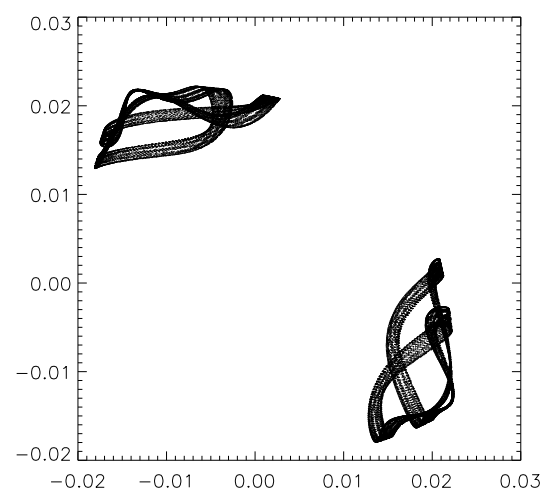

(c)

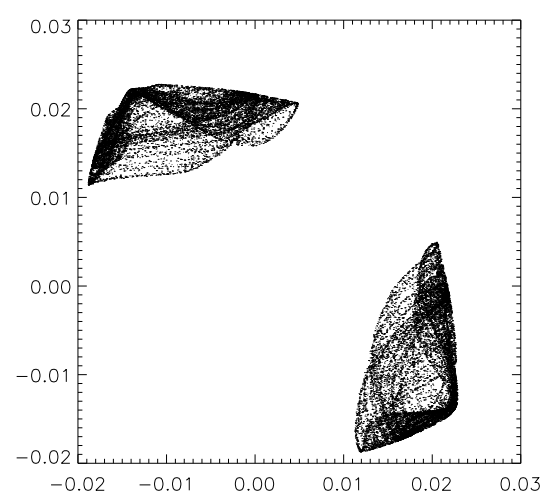

(e)

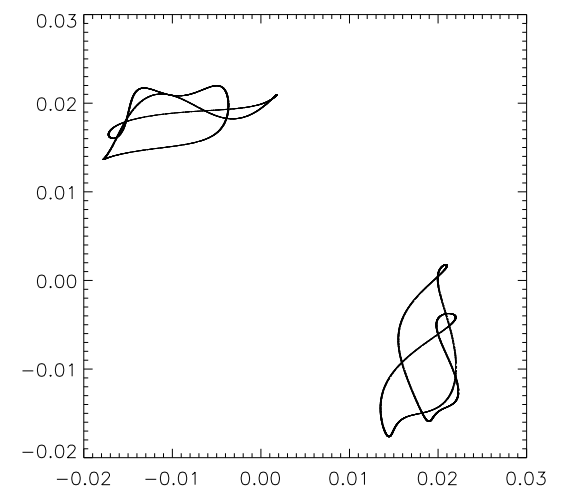

(b)

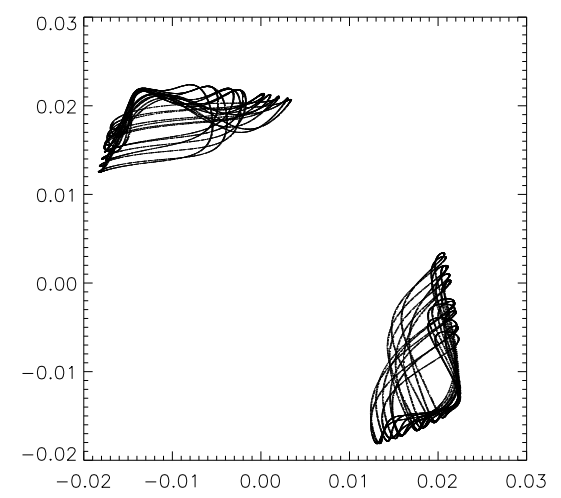

(d)

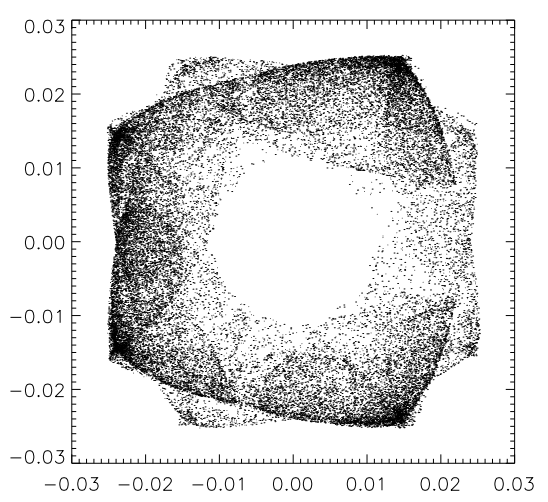

(f)

Figure 10: Poincaré section $q_{1}(\mathbf{v}(t))=0$ of attractors for $R=13.1$ (a), $R=13.117$ (b), $R=13.1175$ (c), $R=13.118$ (d), $R=13.12$ (e) and $R=13.14$ (f). Horizontal axis: $q_{2}(\mathbf{v}(t))$, vertical axis: $q_{3}(\mathbf{v}(t))$. 


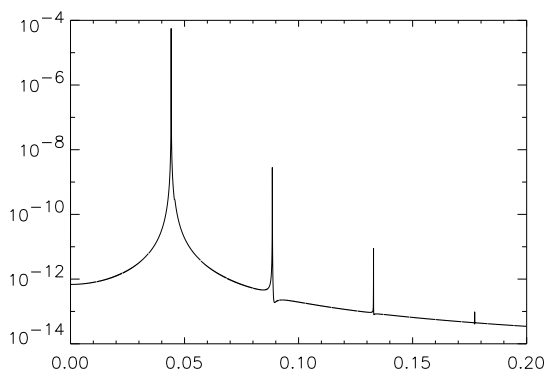

(a)

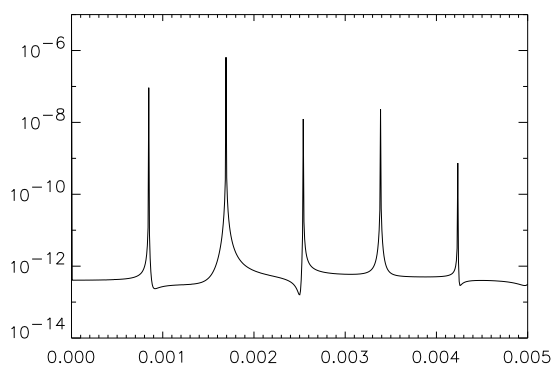

(c)

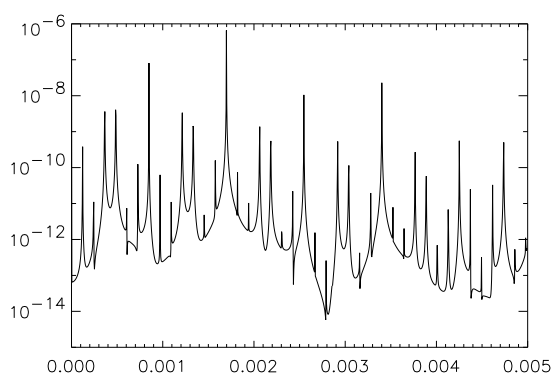

(e)

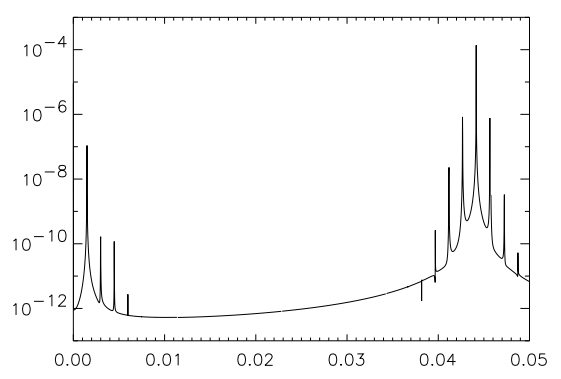

(b)

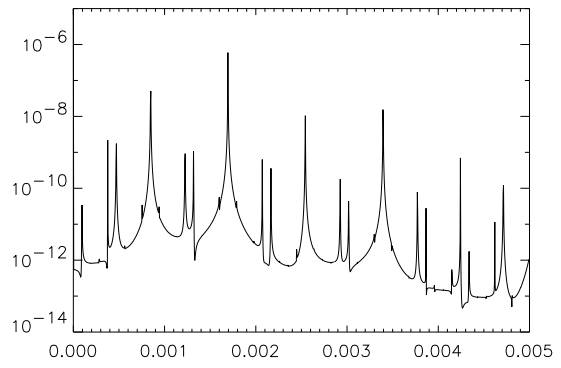

(d)

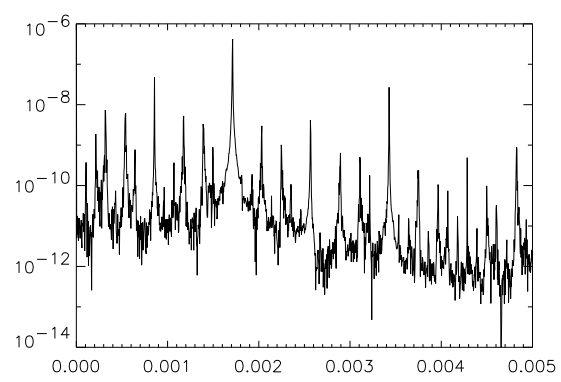

(f)

Figure 11: Frequency spectrum of $q_{1}(\mathbf{v}(t))$ for $R=13.05$ (a), $R=13.1$ (b), $R=13.117$ (c), $R=13.1175$ (d), $R=13.118$ (e) and $R=13.12$ (f). Horizontal axis: frequency (Hz). 
the $S_{1}$ symmetry. Figure 10a, showing a Poincaré section of the attractor, also suggests that the attractor is a torus.

The next bifurcation at $R=R_{2}\left(13.1<R_{2}<13.11\right)$ is torus doubling with emergence of the frequency $f_{2} / 2$ (cf. Figures 10a,b, Figures 11b,c). In contrast the well-known Feigenbaum scenario for period doubling of periodic orbits, torus doubling bifurcation sequences usually terminate after a few doublings [1] and in our case we get transition to a 3 -torus (possessing three main frequencies; see Figures 10c,11d) takes place at $R=R_{3}$ $\left(13.117<R_{3}<13.1175\right)$. At $R=13.1175$, close to the point of bifurcation, the emerging frequency $f_{3}$ is very close (but not exactly equal) to $f_{2} / 18 . f_{3}$ varies with $R$ much faster than $f_{1}$ and $f_{2}$ (see Figures 11d-f). Attractors found in computations are: $R=13.1177$ - a 3-torus; $R=13.118$ - a 2-torus with the main frequencies $f_{1}$ and $f_{2} / 14$ (see Figures 10d,11e); $R=13.119$ - a 2-torus with the main frequencies $f_{1}$ and $f_{2} / 10 ; R=13.1195$ - a 2-torus with the main frequencies $f_{1}$ and $f_{2} / 16 ; R=13.12$ and 13.13 - chaotic (see Figures $8 \mathrm{c}, 10 \mathrm{e}, 11 \mathrm{f})$.

These results are obtained with the resolution of $16^{3}$ harmonics. When the resolution is increased to $24^{3}$ harmonics the sequence of bifurcations is not affected, but the values of $R$ at which the bifurcations occur slightly change: the torus doubling takes place at $13.12<R<13.125 ;$ the transition to the 3 -torus - at $13.125<R<13.13$, for $R=13.13$ the emerging third frequency remains close to $f_{2} / 18$; for $R=13.14$ the behavior is chaotic, resembling the dynamics for $R=13.12$ with the $16^{3}$ resolution.

\subsection{Bifurcation to fully symmetric chaos}

At $R=R_{4}\left(13.13<R_{4}<13.14\right)$ the eight symmetrically related chaotic attractors join into a single attractor possessing on average all symmetries of the system - a symmetry increasing bifurcation in the terminology of [13]. At $R=13.14$ the behaviour becomes intermittent: a trajectory of the system spends a long time (from 2000 to 150,000 time units in a sample run of duration of 1.5 million time units) in the vicinity of one of the former chaotic attractors, then moves to the vicinity of a symmetric copy of the former attractor, the transition taking approximately 1000 time units. As indicated above, the former attractors are labelled by signs of time averages of the quantities $q_{i}$. Computations show, that labels of successive former attractors visited by a trajectory can differ in only one place (i.e. we observe transitions from $(+++)$ to $(++-),(+-+)$ or to $(-++)$, 
but never to, say, $(--+)$ or $(---))$. Comparison of Poincaré sections (Figures 10e,f) also makes it evident that the attractor under consideration is a union of eight former attractors. (See on Figure 8d behaviour of $q_{1}$ near the point of transition from the $(--+$ ) former attractor to the $(+-+)$ one.)

For $13.14 \leq R \leq 13.4$ in the vicinity of the trivial steady state the system possesses the single chaotic attractor having all the symmetries of the system. As the Reynolds number is increased, the average time spent by a sample trajectory in the vicinity of each former attractor decreases. For $R \geq 13.2$ there is no intermittency any more, the solution is chaotic revealing apparent fast mixing within phase space. We can neither identify any bifurcation in this interval, nor can we guarantee absence of bifurcations.

\subsection{Interpretation of the sequence of bifurcations}

We suggest the following explanation of the observed sequence of attractors. When $R$, the only parameter at our disposal, is varied, the eight branches of periodic attractors with the symmetry group $\Sigma(A)=\mathbf{Z}_{3}$ (as well as many other unstable branches) emerge in a generic Hopf bifurcation at $R=R_{0}$. On varying $R$ we also alter the third order normal form coefficients and this results in a secondary bifurcation at $R=R_{1}$, which is a supercritical Hopf bifurcation (or Sacker-Neimark bifurcation) to a quasiperiodic attractor (also with $\left.\Sigma(A)=\mathbf{Z}_{3}\right)$. Note in particular that the frequency introduced in such a secondary Hopf bifurcation must be very small, since near bifurcation all non-trivial solutions of an $\mathbf{S}^{1}$ commuting vector field are periodic and the higher order terms will cause only a slow drift between these orbits.

This secondary bifurcation creates a branch that is however subject to instabilities brought about by the presence of $\mathbf{S}^{1}$ symmetry breaking terms. The terms are apparently responsible for the torus doubling bifurcation at $R=R_{2}$ and also for the Hopf bifurcation at $R=R_{3}$. Both torus doubling and transition from a $T^{n}$-torus to $T^{(n+1)}$ have been investigated analytically (see [9], [30] and references therein). The third frequency that appears varies fast with $R$ and consequently more 2-tori and periodic orbits emerge due to frequency locking on the 3 -torus.

A detailed explanation for the quantitative details of this sequence of bifurcations is not yet available. In particular, the normal form with $\mathbf{S}^{1}$ symmetry cannot model the three frequency quasiperiodicity, and hence why we observe this particular sequence 
of bifurcations. Moreover, when general torus bifurcations are investigated analytically, usually the dynamics of the system cannot be determined in a certain region of the parameter space (Chenciner bubbles) [34]. In our case one can therefore conjecture that for $R>R_{1}$ there exists a 'fat fractal' set of parameters with positive measure on which there are quasiperiodic attractors; within the gaps of this set there are resonances of non-quasiperiodic attractors and complicated bifurcation sequences can occur. Thus the sequence of bifurcations is likely to be more complex than the one outlined in the previous section; due to limitations of numerical investigation we have not attempted to locate and identify all bifurcations.

The transition at $R=R_{4}$ to fully symmetric chaos is a crisis where unstable invariant sets on a basin boundary merge with an attractor to create a more symmetric attractor [13]. It is very similar in character to another secondary bifurcation, also observed in the normal form from $\mathbf{Z}_{3}$ to full symmetry (see Figure 7 ). In the normal form we observe a transition from a $\mathbf{Z}_{3}$ quasiperiodic attractor via a $\mathbf{Z}_{4}$ quasiperiodic attractor to fully symmetric chaos that at first is highly intermittent. These transitions occur under a small change in the normal form coefficient in the presence of the normal form terms breaking the $\mathbf{S}^{1}$ symmetry. This sequence could be truncated to the one observed at $R=R_{4}$.

\section{Discussion}

We have presented a detailed investigation of the generic Hopf bifurcation with the symmetry $\mathbf{O}$ acting as rotations of a cube for an irreducible representation on $\mathbf{C}^{3}$. We consider a general system defined by the third order normal form and a particular hydrodynamical system that has this group of symmetries. For the generic bifurcation problem we classify the possible primary branches and their stability and among other things, find the possibility of direct bifurcation to fully symmetric chaos or to tori and show that the periodic orbit branching is determined by the third order truncation of the normal form.

The third order normal form exhibits a rich variety of bifurcations because of the available dimensions; this is reflected in the secondary bifurcations of the hydrodynamical system. The normal form can be used to analytically explain the first two bifurcations of the hydrodynamical system: emergence of periodic orbits and their transition to eight symmetrically related stable tori; for each of the orbits and of the tori with $\Sigma(A)=\mathbf{Z}_{3}$. 
These bifurcations are followed by torus doubling and appearance of the third frequency; it becomes progressively more difficult to identify subsequent bifurcations. The sequence is concluded with a symmetry increasing bifurcation where eight attractors, each possessing a symmetry group $\Sigma(A)=\mathbf{Z}_{3}$, join into one with full symmetry, $\Sigma(A)=\mathbf{O}$. (Note that all attractors $A$ observed in numerical simulations with $R>R_{0}$ have point symmetry $T(A)$ that is trivial.) This is also reproduced in the normal form. Although this occurs over a small range of Reynolds numbers, we see that many different dynamical behaviours occur within this range.

Neither analytical nor numerical approaches currently provide an explanation of all bifurcations occurring in the hydrodynamical system. This suggests an approach for a further analysis: we are in the process of obtaining the relevant normal form coefficients for the Hopf bifurcation of the hydrodynamical system to enable us to numerically investigate the details of the bifurcations. A paper is currently in preparation [41] which confirms the observed primary bifurcation for the simulations discussed here.

Further analysis of normal form related to Hopf bifurcation with symmetry $\mathbf{O}$ would be helpful, e.g. investigation of secondary bifurcations of the observed branches of periodic solutions. It is of interest to establish either existence of robust heteroclinics between invariant sets in the system defined by the normal form, or to rule them out. The unfolding of the bifurcation by adding symmetry breaking terms, say from $\mathbf{O}$ to $\mathbf{D}_{4}$ and then to no symmetry, could further aid to understand the primary instability of $\mathrm{ABC}$ flows for the more general cases $A=B \neq C$ and $A \neq B \neq C$.

Investigation of bifurcations of the solutions of the Navier-Stokes equations for Reynolds numbers larger than those considered here is presumably possible by the study of interaction of the steady state and Hopf bifurcations; both of these are identified in [37, 38, 39].

The ABC flow instabilities we examine here are restricted to perturbations that have the same spatial periodicity as the flow. As it was proven analytically in $[32,33,36]$, the flow is unstable to long-wavelength perturbations for $R \gg 1$. It is of interest to identify the resultant sequence of bifurcations; however, this task is beyond the scope of this paper. 


\section{Acknowledgements}

PA would like to thank EPSRC grant GR/N14408 for support of this research. The work was initiated during a two month visit of OP to the School of Mathematical Sciences, University of Exeter, UK, from 4 January to 3 March 2001, which was financed by the Royal Society. PA would also like to thank Hinke Osinga for assistance with the xppaut calculations. We are grateful to Paul Matthews for discussions and to the referees for their perceptive comments. A part of numerical results were obtained using computational facilities provided by the program "Simulations Interactives et Visualisation en Astronomie et Mécanique (SIVAM)" at Observatoire de la Côte d'Azur and by the Département Sciences Physiques pour l'Ingénieur of CNRS, France.

\section{References}

[1] A. Arnédo, P. H. Coullet and E. Spiegel. Cascade of period doublings of tori. Phys. Lett. A 94:1-6 (1983).

[2] V.I. Arnold. Sur la topologie des écoulements stationnaires des fluides parfaits. Comptes Rendus Acad. Sci. Paris 261:17-20 (1965).

[3] V.I. Arnold. The evolution of a magnetic field under the influence of transport and diffusion. Some Questions of Present-day Analysis Editor: V M Tikhomirov, Moscow University, 1984. (In Russian.)

[4] V.I. Arnold and E.I. Korkina. The growth of a magnetic field in a three-dimensional incompressible flow. Vestnik Moscow State Univ., Ser. Math. 3, 43-46 (1983) (in Russian).

[5] P. Ashwin. Chaotic intermittency of patterns in symmetric systems. Proceedings of IMA workshop on pattern formation in coupled and continuous systems Editors: $\mathrm{M}$ Golubitsky, D Luss and S H Strogatz, IMA volumes in Mathematics and its applications 115, Springer, 1999.

[6] P. Ashwin and P. Chossat. Attractors for robust heteroclinic cycles with continua of connections. J. Nonlinear Sci. 8, 103-129 (1998). 
[7] P. Ashwin and J. Montaldi. Group theoretic conditions for existence of robust relative homoclinic trajectories. Math. Proc. Camb. Phil. Soc. 133, 125-141 (2002).

[8] T.K. Callahan and E. Knobloch. Symmetry breaking bifurcations on cubic lattices. Nonlinearity 10, 1179-1216 (1997).

[9] A. Chenciner and G. Iooss Bifurcation de Tores invariants. Arch.Rat.Mech.Anal. 69, 109-198 (1979).

[10] S. Childress. New solutions of the kinematic dynamo problem. J. Math. Phys. 11, 3063-3967 (1970).

[11] S. Childress and A.D. Gilbert. Stretch, twist, fold: the fast dynamo, Springer-Verlag, Berlin (1995).

[12] S. Childress and A.M. Soward. On the rapid generation of magnetic field. In Chaos in astrophysics (ed. J.R. Buchler), 233-244 (1985).

[13] P. Chossat and M. Golubitsky. Symmetry increasing bifurcation of chaotic attractors. Physica D 32, 423-436 (1988).

[14] A.P. Dias and I.N. Stewart. Hopf bifurcation on a simple cubic lattice. Dynamics Stab Systems 14, 3-55 (1999).

[15] B. Dionne, M. Golubitsky and I. Stewart, Coupled cells with internal symmetry: II. direct products. Nonlinearity 9, 575-599 (1996).

[16] T. Dombre, U. Frisch, J.M. Greene, M. Hénon, A. Mehr and A. Soward. Chaotic streamlines in the ABC flows. J. Fluid Mech. 167, 353-391 (1986).

[17] G.B. Ermentrout. xppaut (dynamical systems software). Available from http://www . math.pitt.edu/ ${ }^{\sim}$ bard/bardware/ (2000).

[18] B. Galanti, P.L. Sulem and A. Pouquet. Linear and non-linear dynamos associated with ABC flows. Geophys. Astrophys. Fluid Dynamics 66, 183-208 (1992).

[19] B. Galanti, P.L. Sulem and A. Pouquet. Influence of the period of an ABC flow on its dynamo action. In Solar and Planetary Dynamos, Proceedings of a NATO Advanced Study Institute held at the Isaac Newton Institute, Cambridge, September 1992 (eds. 
M.R.E. Proctor, P.C. Matthews \& A.M. Rucklidge), Cambridge Univ. Press, 99-103 (1993).

[20] D.J. Galloway and U. Frisch. A numerical investigation of magnetic field generation in a flow with chaotic streamlines. Geophys. Astrophys. Fluid Dynamics 29, 13-18 (1984).

[21] D.J. Galloway and U. Frisch. Dynamo action in a family of flows with chaotic streamlines. Geophys. Astrophys. Fluid Dynamics 36, 53-83 (1986).

[22] D.J. Galloway and U. Frisch. A note on the stability of a family of space-periodic Beltrami flows. J. Fluid Mech. 180, 557-564 (1987).

[23] A.D. Gilbert. Fast dynamo action in a steady chaotic flow. Nature 350, 483-485 (1991).

[24] A.D. Gilbert. Magnetic field evolution in steady chaotic flows. Phil. Trans. Roy. Soc. London 339, 627-656 (1992).

[25] M. Golubitsky and I.N Stewart. Hopf bifurcation in the presence of symmetry, Arch. Rat. Mech. Anal. 87, 107-165 (1985).

[26] M. Golubitsky, I.N Stewart, and D. Schaeffer. Groups and Singularities in Bifurcation Theory. Volume 2. Appl. Math. Sci. 69, Springer-Verlag, New York, 1988.

[27] J. Guckenheimer and P. Holmes. Structurally stable heteroclinic cycles. Math. Proc. Camb. Phil. Soc. 103, 189-192 (1988).

[28] J. Guckenheimer et al. dstool_tk, available from Cornell at www.cam. cornell.edu.

[29] M. Hénon. Sur la topologie des lignes de courant dans un cas particulier. Comptes Rendus Acad. Sci. Paris 262, 312-314 (1966) (in French).

[30] G. Iooss and J.E. Los. Quasi-Genericity of Bifurcations to High Dimensional Invariant Tori for Maps. Commun.Math.Phys. 119, 453-500 (1988).

[31] I. Klapper and L.S. Young. Rigorous bounds on the fast dynamo growth rate involving topological entropy. Comm. Math. Phys. 173, 623-646 (1995). 
[32] A. Libin and G. Sivashinsky. Long wavelength instability of the ABC flow. Quartely of Appl. Math. XLVIII, 611-623 (1990).

[33] A. Libin, G. Sivashinsky and E. Levich. Long wavelength instability of periodic flows at large Reynolds numbers. Phys. Fluids 30, 2984-2986 (1987).

[34] J.E. Los. Non-normally hyperbolic invariant curves for maps in $R^{3}$ and doubling bifurcation. Nonlinearity 2, 149-174 (1989).

[35] I.S. Melbourne. A singularity theory analysis of bifurcation problems with octahedral symmetry. Dyn. Stab. Systems 4, 293-321 (1986).

[36] H.K. Moffatt. Magnetostatic equilibria and analogous Euler flows of arbitrary complex topology. Part 2. Stability considerations. J. Fluid Mech. 166, 359-378 (1986).

[37] O. Podvigina and A. Pouquet. On the non-linear stability of the 1:1:1 ABC flow. Physica D 75, 471-508 (1994).

[38] O.M. Podvigina. Spatially-periodic steady solutions to the three-dimensional NavierStokes equation with the ABC-force. Physica D 128, 250-272 (1999).

[39] O.M. Podvigina. Evolutionary and steady solutions to the Navier-Stokes equation with the $A B C$ forcing (in Russian), Institute of Mechanics, Lomonosov Moscow State University (1999).

[40] O.M. Podvigina. A route to magnetic field reversals: an example of an ABCforced non-linear dynamo. Submitted to Geophys. Astrophys. Fluid Dynamics [http://xxx.lanl.gov/abs/physics/0107029].

[41] O. Podvigina and P. Ashwin. Center manifold reduction for a Hopf bifurcation with cubic symmetry. In preparation (2002).

[42] M. Silber and E. Knobloch, Hopf bifurcation on a square lattice. Nonlinearity 4, 1063-1106 (1991).

[43] J.W. Swift. Hopf bifurcation with the symmetry of the square. Nonlinearity, 1, 333-377 (1988). 
[44] J.W. Swift and E. Barany. Chaos in the Hopf bifurcation with tetrahedral symmetry; convection in a rotating fluid with low Prandtl number. Euro. J. Mech. B/Fluids 102, 99-104 (1991).

[45] M.M. Vishik. On magnetic field generation by a three-dimensional steady flow of conducting fluid at high magnetic Reynolds number. Izvestia Acad. Sci. USSR, Fizika Zemli N3, 3-12 (1988) (in Russian).

[46] M.M. Vishik. Magnetic field generation by the motion of a highly conducting fluid, Geophys. Astrophys. Fluid Dynamics 48, 151-167 (1989). 